\title{
RESEARCH
}

\section{Link weights recovery in heterogeneous information networks}

\author{
Hong-Lan Botterman ${ }^{1 *}$ and Robin Lamarche-Perrin ${ }^{2}$
}

*Correspondence:
hong-lan.botterman@lip6.fr
'Sorbonne Université, CNRS,
LIP6, 75005 Paris, France
Full list of author information is
available at the end of the article

Springer

\begin{abstract}
Socio-technical systems usually consist of many intertwined networks, each connecting different types of objects or actors through a variety of means. As these networks are co-dependent, one can take advantage of this entangled structure to study interaction patterns in a particular network from the information provided by other related networks. A method is, hence, proposed and tested to recover the weights of missing or unobserved links in heterogeneous information networks (HIN) —abstract representations of systems composed of multiple types of entities and their relations. Given a pair of nodes in a HIN, this work aims at recovering the exact weight of the incident link to these two nodes, knowing some other links present in the HIN. To do so, probability distributions resulting from path-constrained random walks, i.e., random walks where the walker is forced to follow only a specific sequence of node types and edge types, capable to capture specific semantics and commonly called a meta-path, are combined in a linearly fashion to approximate the desired result. This method is general enough to compute the link weight between any types of nodes. Experiments on Twitter and bibliographic data show the applicability of the method.
\end{abstract}

Keywords: Heterogeneous information network, Random walk, Link weight, Regression model

\section{Introduction}

Networked data are ubiquitous in real-world applications. Examples of such data are humans in social activities, proteins in biochemical interactions, pages of Wikipedia or movies-users from Amazon just to name a few [1,2]. These are abstracted by a network where nodes represent the entities (e.g., individuals or pages) of the examined system whilst (directed) links stand for existing physical or virtual ties between them. Weights can also be put on the links to state, for instance, their importance [3,4]. In some cases, the nodes and/or the links are of different nature. For example, in social activities, the links can reflect online or offline communication or more obviously, in the movie-user case, nodes represent two different objects. Taking these differences explicitly into account in the modeling can only enrich the understanding of the inspected system. Thus, heterogeneous information networks (HIN), abstract representations of systems composed of multiple types of entities and their relations, are good candidates to model such data together with

(c) The Author(s) 2021. This article is licensed under a Creative Commons Attribution 4.0 International License, which permits use, sharing, adaptation, distribution and reproduction in any medium or format, as long as you give appropriate credit to the original author(s) and the source, provide a link to the Creative Commons licence, and indicate if changes were made. The images or other third party material in this article are included in the article's Creative Commons licence, unless indicated otherwise in a credit line to the material. If material is not included in the article's Creative Commons licence and your intended use is not permitted by statutory regulation or exceeds the permitted use, you will need to obtain permission directly from the copyright holder. To view a copy of this licence, visit http://creativecommons.org/licenses/by/4.0/. 
their relations, since they can effectively fuse a huge quantity of information and contain rich semantics in nodes and links.

In the last decade, the heterogeneous information network analysis has attracted a growing interest and many novel data mining tasks have been designed in such networks, such as similarity search, clustering, classification and link prediction [5]. The latter can sometimes refer to the term recovery, in the sense that links already exist but are missing or imperfectly observed in the data. This could be due to sampling or depending on the system under scrutiny, due to node/agent's voluntary decision not to give access to all their data (e.g., online social apps). Whatever the reason, capturing the presence of a link is sometimes not enough sufficient. For instance, in a social network, knowing two individuals are linked does not say anything about the frequency of their communication or the strength of their friendship. Hence, recovering the actual link weight can bring useful information as for instance, in recommendation systems where the weight can be taken for the "rating" a user would give to an item [6,7]. The goal of this work is to recover, for a given pair of nodes in a weighted HIN, the actual incident link weight to these two nodes, knowing some other links present in the HIN.

Link prediction can be related to node similarity problem [8-13]. Indeed, the similarity score between two nodes resulting from a particular function of these two nodes, can be seen as the strength of their connection. Here, this function is related to particular random walks on the graph and so, to the probabilities of reaching one node through different paths, starting from another.

In HIN, most of similarity scores $[14,15]$ are based on the concept of meta-path. In simple terms, this corresponds to a concatenation of node types linked by corresponding link types and the type of a node/link is basically a label in the abstract representation. Meta-paths can be used as a constraint to a classic random walk: the walker is allowed to take only paths satisfying a particular meta-path. These path-constrained random walks have the sensitivity to explicitly take into account different semantics present in HIN. For instance, in a bibliographic network, one can distinguish four types of entities: Authors (A), Papers (P), Venues (V) and Topics (T). Starting from a particular paper, if a walker follows the meta-path PVP, he is likely to end to any another paper published in the same venue than the first. Now, if he follows the meta-path PTP, the ending paper will be about the same topic. Even if the starting and ending papers are the same, the semantics behind may be radically different.

Back to our goal, we can see it as a (linear) regression problem where the aim is to recover the link weight, i.e., a continuous value. This is one step further compared with traditional work that mainly treat this problem as a classification one, where the goal is to infer the presence of a link, or as solely a similarity problem, where no explicit relation is established between the similarity and the weight of the link. This means that the target link weight between a pair of nodes is approximated by a linear combination of probabilities, which results from path-constrained random walks performed on the HIN. These probabilities, thus, translate the fact of being at a particular node starting from another one and are the regressors of the linear regression. The linear combination is constructed step by step (by maximizing a specific function), thus facilitating the understanding of the final model as well as its interpretation. The weighting is, therefore, the result of an optimization problem, and not that of the user's choice, as it is often the case when one wants to integrate several meta-paths into a single score. 
To make recovery tasks, data are commonly split into two sets: training set and test set. The proposed method aims at finding a relevant set of meta-paths together with their coefficient such that the difference between the exact link weight and its approximation is minimized. Obtained coefficients are then tested on the test set to validate the method.

The rest of the paper is organized as follows. In "Preliminary concepts" section, some basic concepts about HIN are presented and the problem statement is exposed. We then review some related work in "Related work" section. "Method" section explains our method and we apply it on empirical data in "Experiments" section. First, in "FIFA WorldCup 2014 Twitter data" section. the method is tested to recover the link weights between entities of different types into Twitter data. Then, in Sec. "Bibliographic Data", it is applied on bibliographic data where the target nodes are of the same type. ${ }^{1}$ We finally conclude and discuss some perspectives in "Summary and discussion" section.

\section{Preliminary concepts}

In this section, we present some concepts of weighted HIN useful for the following and define the "weight recovery" problem. Fig. 1 illustrates this section.

Definition 1 (Weighted directed graph) A weighted directed graph is a 5-tuple $G:=$ $\left(V, E, w, \mu_{s}, \mu_{t}\right)$ with $V$ being the node set, $E$ the link multiset, $w: E \rightarrow \mathbb{R}^{+}$the function that assigns to each link a real non negative weight, $\mu_{s}: E \rightarrow V$ the function that assigns to each link a source node, $\mu_{t}: E \rightarrow V$ the function that assigns to each link a target node.

This concept allows us to introduce the definition of HIN which basically is a weighted directed graph with multiple types of nodes and links.

Definition 2 (Heterogeneous Information Network) A HIN $H:=(G, \mathcal{V}, \mathcal{E}, \phi, \psi)$ is a weighted directed graph $G$ along with $\mathcal{V}$ being the node type set, $\mathcal{E}$ the link type set, $\phi: V \rightarrow \mathcal{V}$ the function that assigns a node type to each node and $\psi: E \rightarrow \mathcal{E}$ the function that assigns a link type to each link such that if two links belong to the same link type, the two links share the same starting and target node type, i.e., $\forall e_{1}, e_{2} \in E,\left(\psi\left(e_{1}\right)=\psi\left(e_{2}\right)\right) \Rightarrow\left(\phi\left(\mu_{s}\left(e_{1}\right)\right)=\phi\left(\mu_{s}\left(e_{2}\right)\right) \wedge \phi\left(\mu_{t}\left(e_{1}\right)\right)=\phi\left(\mu_{t}\left(e_{2}\right)\right)\right)$.

Note also that if two links are of the same type and connect the same two nodes, they are in fact only one with a certain weight.

Fig. 1a illustrates such a network composed of five node types and twenty link types. However, disentangling the different entities present in the HIN is not necessarily a trivial task. Indeed, it sometimes takes a broader view of the system in question to describe it. For that purpose, the concept of network schema, i.e. the meta level description of the HIN, is proposed. In simple terms, this corresponds to the graph defined over the node and link types of the associated HIN. It is represented in Fig. 1b.

Definition 3 (HIN Schema) Let $\mathrm{H}$ be a HIN. The schema $T_{H}$ for $H$ is a directed graph defined on the node types $\mathcal{V}$ and the link types $\mathcal{E}$. Formally, $T_{H}:=\left(\mathcal{V}, \mathcal{E}, v_{s}, v_{t}\right)$ with

\footnotetext{
${ }^{1}$ Compared to the preliminary version presented in [16], this paper confronts the results with other similarity measures present in the literature as discussed in "Using other similarity measures" section (see "Experiments" section), and investigates the applicability of the proposed method on another dataset of a totally different nature and semantics (see in particular Sec."Bibliographic Data"), allowing to refer to other structural objects in graphs, that is to say: motifs.
} 
a

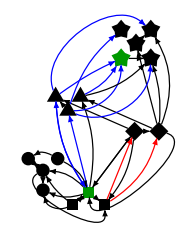

b

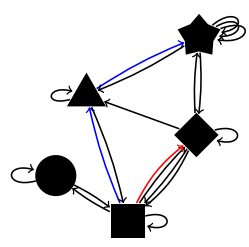

c

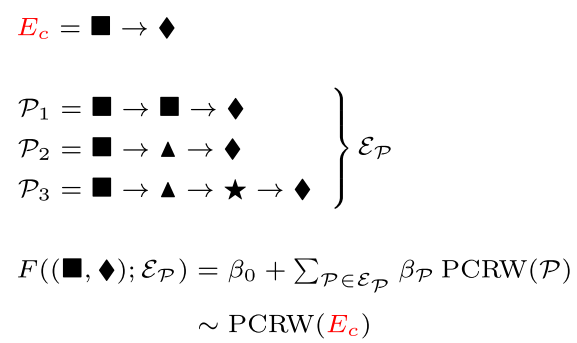

Fig. 1 a Example of HIN composed of multiple node types, represented by diverse shapes, an multiple link types. Nodes are already grouped by shapes. $\mathbf{b}$ Its associated network schema composed by five nodes and twenty links. Each node corresponds to a set of nodes in the corresponding HIN. In the same way, each link is a set of links in the corresponding HIN. See for instance the paths and meta-path of length two in blue

$\boldsymbol{\square} \rightarrow \boldsymbol{\Delta} \rightarrow \star$; the blue paths are said to satisfy the blue meta-path. $\mathbf{c}$ llustration of the problem statement. For each pair of nodes in $(\mathbf{\square}, \mathbf{\vee})$, there is possibly a link connecting them. The link weight is approximated by a linear combination of the path-constrained random walk results, i.e., probability distributions of being at a particular node. Roughly speaking, the probabilities resulting from the random walk constrained by the target meta-path $E_{C}=\mathbf{\square} \rightarrow$, denoted by PCRW $\left(E_{C}\right)$, are expressed as a linear combination $F$ of probabilities resulting from the random walks constrained by three different meta-paths $\mathcal{P}_{1}=\mathbf{\square} \rightarrow \mathbf{\square} \rightarrow$

$\mathcal{P}_{2}=\boldsymbol{\square} \rightarrow \boldsymbol{\Delta} \rightarrow \boldsymbol{\Downarrow} \mathcal{P}_{3}=\boldsymbol{\square} \rightarrow \boldsymbol{\Delta} \rightarrow \star \rightarrow \boldsymbol{\forall}$, denoted by PCRW $\left(\mathcal{P}_{1}\right), \operatorname{PCRW}\left(\mathcal{P}_{2}\right)$ and $\operatorname{PCRW}\left(\mathcal{P}_{3}\right)$,

respectively, whose real-valued coefficients are $\beta_{\mathcal{P}_{1}}, \beta_{\mathcal{P}_{2}}$ and $\beta_{\mathcal{P}_{3}}$, respectively, plus a possible independent term $\beta_{0}$, that is to say $F\left((\mathbf{\square}, \mathbf{\vee}) ; \mathcal{E}_{\mathcal{P}}\right)=\beta_{0}+\sum_{\mathcal{P} \in \mathcal{E}_{\mathcal{P}}} \beta_{\mathcal{P}} P C R W(\mathcal{P})$. One can see that other meta-paths exist between nodes in $(\mathbf{\square}, \mathbf{v})$. The problem is to identify the "best" $\mathcal{E}_{\mathcal{P}}$ and a linear function $F$ with respect to $\operatorname{PCRW}\left(E_{C}\right)$

$v_{s}: \mathcal{E} \rightarrow \mathcal{V}: E^{*} \mapsto v_{s}\left(E^{*}\right):=\phi\left(\mu_{s}(e)\right)$ the function that assigns each link a source node and $v_{t}: \mathcal{E} \rightarrow \mathcal{V}: E^{*} \mapsto v_{t}\left(E^{*}\right):=\phi\left(\mu_{t}(e)\right)$ the function that assigns each link a target node, where $e \in E$ such that $\psi(e)=E^{*}$.

Note that we can effectively take any such element $e \in E$ since $\left\{e \in E \mid \psi(e)=E^{*}\right\}$ is the equivalence class of any of its elements, with the equivalence relation "has the same type of". By definition of HIN, it is sufficient to take one member of the equivalence class to know the node types that the link type $E^{*}$ connects.

Two entities in a HIN can be linked via different paths and these paths have different semantics. These paths can be defined as meta-paths as follows.

Definition 4 (Meta-path [5]) A meta-path $\mathcal{P}$ of length $n \in \mathbb{N}$ is a sequence of node types $V_{0}, \cdots, V_{n} \in \mathcal{V}$ linked by link types $E_{1}, \cdots, E_{n} \in \mathcal{E}$ as follows: $\mathcal{P}=V_{0} \stackrel{E_{1}}{\rightarrow}$ $V_{1} \cdots V_{n-1} \stackrel{E_{n}}{\rightarrow} V_{n}$ which can also be denoted as $\mathcal{P}=E_{1} E_{2} \cdots E_{n}$.

Given a meta-path $\mathcal{P}=V_{0} \stackrel{E_{1}}{\rightarrow} V_{1} \cdots V_{n-1} \stackrel{E_{n}}{\rightarrow} V_{n}$ and a path $P=v_{0} \stackrel{e_{1}}{\rightarrow}$ $v_{1} \cdots v_{n-1} \stackrel{e_{n}}{\rightarrow} v_{n}$, if $\forall i \in\{0, \ldots, n\}, \phi\left(v_{i}\right)=V_{i}, \forall i \in\{1, \ldots, n\}, \mu_{s}\left(e_{i}\right)=v_{i-1}, \mu_{t}\left(e_{i}\right)=v_{i}$ and $\psi\left(e_{i}\right)=E_{i}$, then path $P$ satisfies meta-path $\mathcal{P}$ and we note $P \in \mathcal{P}$. Hence, a meta-path is a set of paths.

One denotes by $\mathcal{P}^{-1}$ the inverse meta-path of $\mathcal{P}$, i.e., $\mathcal{P}^{-1}=V_{n} \stackrel{E_{n}^{-1}}{\longrightarrow} V_{n-1} \cdots V_{1} \stackrel{E_{1}^{-1}}{\longrightarrow}$ $V_{0}$. The link type $E_{i}^{-1}$ is the inverse of $E_{i}$ : when it links the same node types, it is equal to itself, $E_{i}^{-1}=E_{i}$ while when the node types are different, the associated semantic is the inverse in term of "active/passive". 
In Fig. 1b, an example of meta-path is $\mathbf{\square} \rightarrow \boldsymbol{\Delta} \rightarrow \star$, in blue, in the network schema. Blue paths in the HIN in Fig. 1a are said to satisfy this meta-path since each one of their segments respects the aforementioned conditions.

Problem 1 (Weight recovery) Let be a $\operatorname{HIN} H=(G, \mathcal{V}, \mathcal{E}, \phi, \psi)$, with $G=\left(V, E, w, \mu_{s}, \mu_{t}\right)$ a directed and weighted graph, and a target link type $E_{c}$ between two node types. The "weight recovery problem" is to find a set of relevant meta-paths $\mathcal{E}_{\mathcal{P}}$ and a linear function $F$ of probabilities resulting from random walks constrained by these meta-paths that best quantifies, for each pair of nodes in $H$, the strength of their connection via the target link type $E_{c}$.

\section{Related work}

Compared to previous work, which usually focuses on undirected and binary graphs, the approach we present addresses the recovery of directed and weighted links in HIN. To this end, our regression model directly estimates the weight of links without computing any intermediate ranking on these links, or applying any threshold to reduce the recovery task to binary graphs.

As previously explained, our work is based on node similarity measures and thus, is also related to link prediction [8-13]. Similarity measures and link prediction have been extensively studied in the past few years and for a deeper review of them, see $[17,18]$. Nonetheless, one often roughly differentiates two kinds of approaches: unsupervised versus supervised. For the first category, one often proposes different similarity measures based upon either node attributes or the topology of the underlying graph. One can further distinguish local from global indices. Local indices makes use of local neighborhood information, e.g., Adamic-Adar index, Common Neighbor or Preferential Attachment Index, Ressource Allocation just to name a few. By contrast, global indices are based on global properties such as paths. These encompass Shortest Path, Katz or measures using random walks, e.g., Random Walk with Restart, PageRank, Hitting Time, Commute Time and so on. Based on these aforementioned features, a plethora of supervised methods have been conceived to predict links. Amongst them, one distinguishes feature-based classification [19,20] from probabilistic model [21,22] and matrix factorization [23]. However, all these measures are mostly used in homogeneous networks.

Recently, several measures have tackled the problem of node similarity in HIN which takes into account not only the structure similarity of two entities but also the meta-paths connecting them. Amongst these measures, PathCount (PC [15]) and Path Constrained Random Walk (PCRW [14]) are the two most basic and gave birth to several extensions [24-27].

Methods related to $\mathrm{PC}$ are based on the count of paths between a pair of nodes, given a meta-path. PathSim [28] measures the similarity between two objects of same type along a symmetric meta-path which is restrictive since many valuable paths are asymmetric and the relatedness between entities of different types is not useless. Two measures based on it $[29,30]$ incorporate more information such as the node degree and the transitivity. However, all these methods have the drawback of favoring highly connected objects since they deal with raw data.

Methods related to PCRW are based on random walks and so the probability of reaching a node from another one, given a meta-path. Considering a random walk implies a nor- 
malization and, depending on the data, offers better results. An adaptation, HeteSim [31], measures the meeting probability between two walkers starting from opposite extremities of a path, given a meta path. However, this method requires the decomposition of atomic relations for odd-length meta-paths. This decomposition allows the walkers to meet at the middle of the meta-path and at the same node type but it is very costly for large graphs. To address this issue, AvgSim [32] computes the similarity between two nodes using random walks conditioned by a meta-path and its inverse. But it is mostly appreciated in undirected networks since in these cases, it is just as sensible to walk a path in one direction as in the other.

In these cited works, when the similarity scores are used for link prediction/detection, the scores are ranked and then, the presence of links is inferred based on this ranking. Also some work try to (manually) combine meta-paths but the target values to recover are binary; the networks are unweighted, making the problem a classification one. At variance with these works, we set ourselves in the general framework of directed and weighted HINs. We do not use any ranking or threshold but take directly the similarity measures obtained by means of an adequate combination of PCRWs as link weights. This combination is constructed step by step, allowing one to follow its evolution and, hence, facilitating the understanding of the final result. This allows not only to perform description tasks but also, to some extent, recovery tasks.

Other work with similar objectives to those pursued here are those relating to recommendation systems. These are software tools and techniques providing suggestions for items to be of use to a user [33]. They are often based on one of the following two paradigms [34-36]: collaborative filtering [37] or content-based filtering [38]. Due to the richness (e.g., dimension) of many networked data, it is sometimes difficult to use them directly for recommendation (or any other data mining task). Recently, network embedding $[39,40]$, the approach of learning latent low-dimensional feature representations for the nodes or links in a network, has been successfully applied in a myriad of tasks and, in this case, recommendation [36]. However, a disadvantage of node embedding is that we lose some of the interpretability/explanability of the original data, which is a main objective in the present work. Most of the embedding methods focus on homogeneous information netoworks. However, very recently, some work propose methods to analyze heterogeneous networks [41-43]. To combine these ideas, Shi et al. have proposed a heterogeneous network embedding based approach for HIN based recommendation, HERec [44]. Meaningful node sequences for network embedding are generated by a meta-pathbased random walk strategy and are then integrated into an extended matrix factorization method.

\section{Method}

We present our method for solving Problem 1 in three steps. Consider a HIN and let us denote by $E_{c}$ the target link type defined between $V_{0}$ and $V_{n}$. We consider a meta-path $\mathcal{P}=V_{0} \stackrel{E_{1}}{\rightarrow} V_{1} \cdots V_{n-1} \stackrel{E_{n}}{\rightarrow} V_{n}$ different from $E_{c}$. There may be repetitions in this sequence of nodes and links. Let us introduce the notation $\mathcal{P} \equiv \mathcal{P}^{0, n}$ and let us denote by $\mathcal{P}^{a, b}$ the truncated meta-path of $\mathcal{P}$ from node type $V_{a}$ to $V_{b}$. 


\section{Path-constrained random walk}

Let $X_{i} \in V_{i}$ be a random variable representing the position of a random walker in the set $V_{i}$. A random walk starting from $X_{0}$ constrained by the meta-path $\mathcal{P}$ corresponds to a discrete-time Markov chain, i.e., a sequence of random variables $X_{0}, X_{1}, \ldots, X_{n}$ with the Markov property: $\forall i \in\{0, \ldots, n\}, \forall\left(v_{0}, \ldots, v_{n}\right) \in V_{0} \times \ldots \times V_{n}$,

$$
\mathbb{P}\left(X_{i}=v_{i} \mid X_{i-1}=v_{i-1}, \ldots, X_{0}=v_{0}\right)=\mathbb{P}\left(X_{i}=v_{i} \mid X_{i-1}=v_{i-1}\right) .
$$

Here, since there may be more than one link type between two node types, we introduce the notation $\mathbb{P}\left(\left(X_{i}=v_{i} \mid X_{i-1}=v_{i-1}\right) \mid \mathcal{P}^{i, i+1}\right)=: \mathbb{P}\left(\left(v_{i} \mid v_{i-1}\right) \mid \mathcal{P}^{i, i+1}\right)=\mathbb{P}\left(\left(v_{i} \mid v_{i-1}\right) \mid E_{i}\right)$ to emphasize the fact that the random walk is constrained by the meta-path $\mathcal{P}$. This means that for a walker to reach $v_{i}$ from $v_{i-1}$, he has to follow only links of type $E_{i} \equiv \mathcal{P}^{i, i+1}$. The probability $\mathbb{P}\left(\left(v_{i} \mid v_{i-1}\right) \mid E_{i}\right)$, thus, defined is computed as

$$
\mathbb{P}\left(\left(v_{i} \mid v_{i-1}\right) \mid E_{i}\right)=\frac{w_{E_{i}}\left(v_{i-1}, v_{i}\right)}{\sum_{k} w_{E_{i}}\left(v_{i-1}, v_{k}\right)},
$$

where $w_{E_{i}}\left(v_{j}, v_{k}\right)$ is the link's weight of type $E_{i}$ between nodes $v_{j}$ and $v_{k}$, i.e., $w_{E_{i}}\left(v_{j}, v_{k}\right)=$ $w(e)$ such that $e \in E, \psi(e)=E_{i}, \mu_{s}(e)=v_{j}$ and $\mu_{t}(e)=v_{k}$.

Thenceforth, given $v_{n} \in V_{n}$ and $v_{0} \in V_{0}$, the probability of reaching $v_{n}$ from $v_{0}$ following the meta-path $\mathcal{P}$, denoted by $\mathbb{P}\left(\left(v_{n} \mid v_{0}\right) \mid \mathcal{P}\right)$, is simply defined by the random walk starting at $v_{0}$ and ending at $v_{n}$ following only paths satisfying $\mathcal{P}$. This conditional probability may be expressed recursively (and by making use of eq. (1)) by means of the law of total probability

$$
\begin{aligned}
\mathbb{P}\left(\left(v_{n} \mid v_{0}\right) \mid \mathcal{P}\right) & =\sum_{v_{n-1} \in V_{n-1}}\left[\mathbb{P}\left(\left(v_{n} \mid v_{n-1}\right) \mid E_{n}\right) \times \mathbb{P}\left(\left(v_{n-1} \mid v_{0}\right) \mid \mathcal{P}^{0, n-1}\right)\right] \\
& =\sum_{v_{n-1} \in V_{n-1}}\left[\frac{w_{E_{n}}\left(v_{n-1}, v_{n}\right)}{\sum_{k} w_{E_{n}}\left(v_{n-1}, v_{k}\right)} \times \mathbb{P}\left(\left(v_{n-1} \mid v_{0}\right) \mid \mathcal{P}^{0, n-1}\right)\right],
\end{aligned}
$$

with $\mathbb{P}\left(\left(v_{1} \mid v_{0}\right) \mid \mathcal{P}^{0,1}\right)=w_{E_{1}}\left(v_{0}, v_{1}\right) / \sum_{k} w_{E_{1}}\left(v_{0}, v_{k}\right)$ the basis of recurrence. In the following, we use the notation $\operatorname{PCRW}(\mathcal{P})$ to denote the column vector of such conditional probabilities $\mathbb{P}\left(\left(v_{n} \mid v_{0}\right) \mid \mathcal{P}\right), \forall v_{0}, v_{n}$.

For instance, in the HIN in Fig. 1a, the probability for a walker to reach the green star $\star$ from the green square $\square$ following the meta-path $\mathbf{\square} \rightarrow \boldsymbol{\Delta} \rightarrow \star$ equals $5 / 12$.

Note that we forbid the walker to return to the initial node on the penultimate step of the walk, i.e., if $V_{n-1}=V_{0}$, the sum in eq. (2) only holds for all $v_{n-1} \neq v_{0}$. When training the model, we indeed need to remove from the data the pieces of information (links) we aim to predict, before actually applying and evaluating the prediction scheme. In other words, a link cannot be used to predict itself.

Remark 1 (Hole nodes) It is possible that a node $v_{i} \in V_{i}$ is not connected to any node $v_{j} \in$ $V_{j}$ by the link type $E_{i j}$ and thus, the transition probability is not defined. To overcome this problem, we provide each set $V_{k}$ with a hole node $h_{k}$ on which point all the disconnected nodes. Plus, all the holes are connected with each other and holes cannot point to another node (i.e., no hole node). Formally, $\forall V_{k} \in \mathcal{V}, V_{k}^{h}:=V_{k} \cup\left\{h_{k}\right\} . \forall E_{i j} \in \mathcal{E}$, if $w_{E_{i j}}\left(v_{i}, v_{j}\right)=$ $0, \forall v_{j} \in V_{j}$ then $w_{E_{i j}}\left(v_{i}, h_{j}\right)=1$, otherwise $w_{E_{i j}}\left(v_{i}, h_{j}\right)=0$. Furthermore, $\forall E_{i j} \in \mathcal{E}$, $w_{E_{i j}}\left(h_{i}, h_{j}\right)=1$ and $\sum_{v_{j} \in V_{j}} w_{E_{i j}}\left(h_{i}, v_{j}\right)=0$. In this fashion, transition probabilities are always well defined. 


\section{Linear regression model}

Since $H$ is a HIN, multiple types of links can connect the nodes. Hence, there is no reason to restrict ourselves to a single meta-path to compute the reachability of one node from another. As a result, the similarity between $v_{n}$ and $v_{0}$ is defined by several path-constrained random walk results combined through a linear regression model of the form

$$
F\left(\left(v_{n} \mid v_{0}\right) \mid \mathcal{E}_{\mathcal{P}}\right):=\beta_{0}+\sum_{\mathcal{P} \in \mathcal{E}_{\mathcal{P}}} \beta_{\mathcal{P}} \mathbb{P}\left(\left(v_{n} \mid v_{0}\right) \mid \mathcal{P}\right)
$$

where $\mathcal{E}_{\mathcal{P}}$ is the set of selected meta-paths and the vector $\beta:=\left[\beta_{0}, \beta_{1}, \cdots, \beta_{\left|\mathcal{E}_{\mathcal{P}}\right|}\right]^{\mathrm{T}}$ is realvalued coefficients. The coefficients stress the contribution of each meta-path in the final similarity score $F\left(\left(v_{n} \mid v_{0}\right) \mid \mathcal{E}_{\mathcal{P}}\right)$. Since the components of $\beta$ are not confined in $[0,1]$ and do not sum to 1 , i.e., not a convex combination, $F$ is a real-valued function whose image is neither confined in $[0,1]$.

Now, we have a linear regression problem since we want to recover the exact link weights with respect to $E_{c}$. The dependent variable is, thus, $\operatorname{PCRW}\left(E_{c}\right)$ whilst the predictors are $\operatorname{PCRW}(\mathcal{P}), \mathcal{P} \in \mathcal{E}_{\mathcal{P}}$. The choice of linear model is simply motivated by its interpretability in our particular case. Given example node pairs and their link weights, $\beta$ is estimated by the least squares method which is appreciated for its applicability and simplicity. In formulae, with $\mathbb{1}$ the column vector whose entries are 1 :

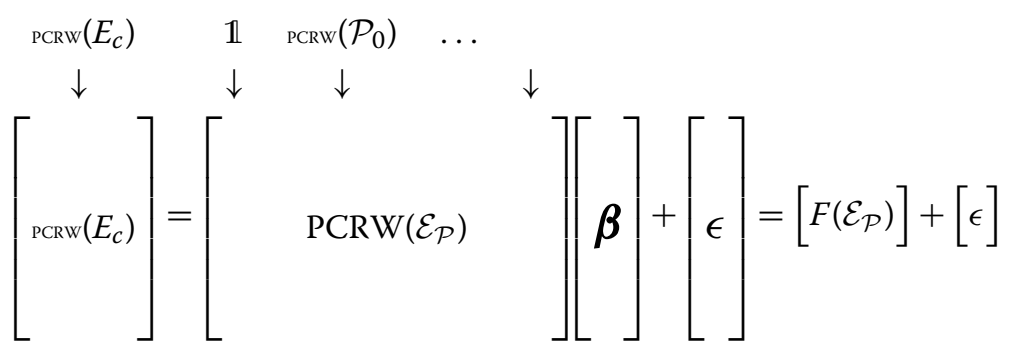

and we choose $\hat{\beta}$ such that the residual sum of squares RSS $=\epsilon^{T} \epsilon=\|\epsilon\|^{2}$ is minimized.

\section{Forward selection procedure}

To determine the set $\mathcal{E}_{\mathcal{P}}$, we use the forward selection with $p$-value and $r^{2}$ criteria. This is a greedy approach but very simple and intuitive. The $p$-values are used to test the significance of each predictor. Given the hypothesis $H_{0}: \beta=0$ against the hypothesis $H_{1}: \beta \neq 0$, the $p$-value $p$ is the probability, under $H_{0}$, of getting a statistics as extreme as the observed value on the sample. We reject the hypothesis $H_{0}$, at the level $\alpha$, if $p \leq \alpha$ in favor of $H_{1}$. Otherwise, we reject $H_{1}$ in favor of $H_{0}$. Conversely, the $r^{2}$ score is used to test the quality of the entire model. It is the proportion of the variance in the dependent variable that is predictable from the predictors. Note that the $r^{2}=1$-RSS/TSS where TSS is the total sum of squares, i.e., is the sum of the squares of the difference of the dependent variable and its mean. Hence, maximizing the $r^{2}$ is equivalent to minimizing the RSS.

So, given $k$ predictors or explanatory variables which are the probability distributions $\operatorname{PCRW}\left(\mathcal{P}_{k}\right)$, the forward selection procedure works as follows

- Start with a null model, i.e., no predictor but only an intercept. Typically, this is the average of the dependent variable; 
- Try $k$ linear regression models (i.e., models with only one predictor) and chose the one which gives the best model with respect to the criterion. In our case, the one that minimizes RSS or alternatively, the one that maximizes the coefficient of determination $r^{2}$

- Search among the remaining variables the one that, added to the model, gives the best result, i.e., the higher $r^{2}$ such that all the variables in the model are significant, i.e., their $p$-value is below the chosen threshold. Iterate this step until no further improvement.

Note that by proceeding in this way, and contrary to meta-path fusion methods such as in [44], we can easily check the significance of each explanatory variable, which ultimately means testing the significance of each meta-path in the final model, not just their weights. Finally, it should be mentioned that this method contains very few parameters, which is appreciable since parameter tuning always depends on the data under consideration.

\section{Validation}

Since we would like to use the regression model as a prediction model (i.e., not only a descriptive one), we use Monte Carlo cross-validation a.k.a. repeated random subsampling validation [45]. Given a dataset of $N$ points, the method simply splits them into a training subset $s_{t}$ and a test subset $s_{v}$. The model is then trained on $s_{t}$ and tested on $s_{v}$. This procedure is repeated multiple times and the results are then averaged over the splits. Note that the results of Monte Carlo cross-validation tend towards those of leave- $p$ out cross-validation [46] as the number of random splits tends to infinity. The drawbacks of this method are the possibility that some observations may never be selected for training or on the contrary, may be used at each split. Plus, the results depend on the different random splits, i.e., it displays Monte Carlo variation. However, it has advantage (over $k$-fold cross validation [46]) as the proportion of the split is independent of the folds (iterations). It means Monte Carlo allows to explore somewhat more possible partitions, though one is unlikely to get all of them since there exist $C_{N}^{S_{t}}$ unique training subsets.

Remark 2 (Division of a node type) Given a HIN $H$ with $\mathcal{V}=\left\{V_{1}, \ldots, V_{k}, \ldots, V_{m}\right\}$ the set of node types with $V_{k}=\left\{V_{k, 1}, \ldots, V_{k, q}\right\}$, one can want to understand the "role" of each $V_{k, r}$. Let two node types $V_{i}$ and $V_{j}$ (not necessarily distinct) be the target node types and $\mathcal{E}_{\mathcal{P}}$ the set of meta-paths. Plus, let $V_{i}$ and $V_{j}$ be linked by a specific meta-path including the node type $V_{k}$, namely, $\mathcal{P}=V_{i} \cdots \stackrel{e_{k}}{\rightarrow} V_{k} \cdots \stackrel{e_{j}}{\rightarrow} V_{j}$ with $\mathcal{P} \in \mathcal{E}_{\mathcal{P}}$. We can, thus, construct $q$ subsets $S_{i, r}=\left\{v_{i} \in V \mid \phi\left(v_{i}\right) \in V_{i} \wedge \exists P=v_{i} \cdots \stackrel{e_{k}}{\rightarrow} v_{k, r} \cdots \stackrel{e_{j}}{\rightarrow} v_{j}\right\}$ and $q$ subsets $S_{j, r}=\left\{v_{j} \in V \mid \phi\left(v_{j}\right) \in V_{j} \wedge \exists P=v_{i} \ldots \stackrel{e_{k}}{\rightarrow} v_{k, r} \cdots \stackrel{e_{j}}{\rightarrow} v_{j}\right\}(r=1, . ., q)$ such that with $v_{k, r} \in V_{k, r} \subseteq V_{k}\left(v_{j} \in V_{j}\right.$ and $v_{i} \in V_{i}$ resp.) and $P \in \mathcal{P}$. We can, thus, build $q$ linear regression models: one for each HIN $H_{r}$ formed from the node set $\left\{v \in V \mid \phi(v) \in \mathcal{V} \backslash\left\{V_{k}, V_{i}, V_{j}\right\}\right\} \cup\left\{S_{i, r}, S_{j, r}\right\}$ with meta-paths $\mathcal{E}_{\mathcal{P}} \backslash \mathcal{P}$. Analyzing the vector $\hat{\beta}$ of each final model can bring some insight about the "role" of each $V_{r}$.

\section{Using other similarity measures}

Until now, we directly take the probabilities resulting from random walks to compute the similarity between nodes (eq. (2)). However, this is only one possible example of similarity measure and many others could be used within the same framework. In particular, one can 
cite the following two measures, often used for comparison in other works and appreciated for their wide applicability (see Sec. for a more extensive review of the literature on this matter):

- Path Count (PC [15]): instead of computing the probability (i.e., normalizing), one only considers the number of paths linking two nodes. In our case, we consider the weighted paths. More precisely $\operatorname{PC}\left(\left(v_{i} \mid v_{i-1}\right) \mid E_{i}\right)=w_{E_{i}}\left(v_{i-1}, v_{i}\right)$, and eq. (2) is modified according to this. Note however it is no longer a probability and so, eq. (2) is rather $\mathrm{PC}\left(\left(v_{n} \mid v_{0}\right) \mid \mathcal{P}\right)$;

- AvgSim (AS [32]): it is the average of two probabilities from two path-constrained random walks where paths are the opposite of each other: $\operatorname{AS}\left(\left(v_{n} \mid v_{0}\right) \mid \mathcal{P}\right)=$ $\left[\mathbb{P}\left(\left(v_{n} \mid v_{0}\right) \mid \mathcal{P}\right)+\mathbb{P}\left(\left(v_{0} \mid v_{n}\right) \mid \mathcal{P}^{-1}\right)\right] / 2$.

In this fashion, one can replace in the eq. (3), the probability $\mathbb{P}\left(\left(v_{n} \mid v_{0}\right) \mid \mathcal{P}\right)$ by the similarity measures defined just above. Indeed, one can appreciate the generality of the proposed framework by considering other similarity measures.

This way, we want to insist on the fact that the interest of this method is not so much the fact of considering a particular way of calculating the similarity between two nodes but rather its progressive construction of a model which integrates the meta-paths one by one, allowing one to follow the evolution and clearly understand the final result. Plus, the obtained similarities are used to infer the weights of links, not their mere presence or a simple ranking tasks.

In the following, unless stated otherwise, the results are obtained with the simple pathconstrained random walks, i.e., eq. (2). This is motivated by the simplicity of interpretation in terms of probabilities and the analogy with a random surfer, abundantly used in the literature and in many contexts.

\section{Experiments}

We test the proposed methods on two real-world datasets. The first one, related to FIFA WorldCup 2014 Twitter data, allow us to perform tests between target nodes with different types. The task consists in recovering the user-hashtag frequency. The second data set, related to bibliographic data, focus on target nodes of the same types and tackles the problem of co-authorship.

\section{FIFA WorldCup 2014 Twitter data}

We present the dataset on which we test the proposed method as well as the construction of the resulting graphs. Then, we report our results concerning different tests namely, the importance of meta-path length, a description task and eventually a recovery task.

\section{Dataset description and setup}

The data we use are a set of tweets collected from Twitter during the Football World Cup 2014. This period extends from June 12 to July 13, 2014. Twitter allows multiple kinds of interactions between its users. Here, we consider retweet (RT), reply (RP) and mention (MT) actions plus the fact of posting hashtags (UH). The RT relationship means that a user broadcasts a tweet previously posted by another user. The RP action is simply a response tweet to another user in connection with her previous tweet. The last action 


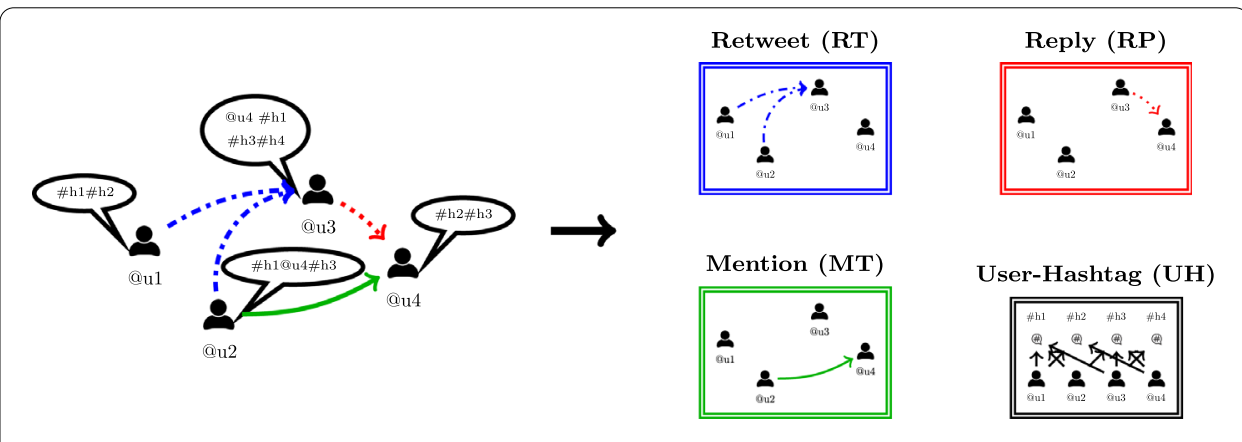

Fig. 2 Illustration of the construction of graphs based on Twitter interactions where four users interact with each other through three types of interactions: retweet RT, reply RP and mention MT; and write some hashtags in their post UH. The underlying HIN is such that $\mathcal{V}=\{$ users, hashtags $\}$ and $\mathcal{E}=\{R T, R P, M T, U H\}$. The four graphs associated with the types of actions are displayed separately for convenience

considered between users is the MT action. This happens when a user mentions explicitly another user in her post. Finally, the UH action links users with their posted hashtags.

Based on these actions, we construct a HIN with two node types $\mathcal{V}=\{$ users, hashtags $\}$ and four edge types $\mathcal{E}=\{\mathrm{RT}, \mathrm{RP}, \mathrm{MT}, \mathrm{UH}\}$ as illustrated in Fig. 2. Each node represents a user or a hashtag. We create a link from $u_{1}$ to $u_{2}$ if $u_{1}$ retweets, replies (to) or mentions $u_{2}$ and the weight of the link correspond to the number of times $u_{1}$ performs the specific action towards $u_{2}$ during the whole world cup. For the user-hashtag graph, a link exists between $u$ and $h$ if $h$ appears in $u$ 's post and the weight of the link corresponds to the number of times $u$ post $h$ during the whole world cup. Note that we exclude hashtags present in the retweeted posts since in these cases, users do not write them themselves. Furthermore, considering them would provoke a trivial correlation between UH and RTUH. All graphs are directed and weighted.

The dataset contains 13,826 users and 14,392 hashtags. The RT graph is composed of 6069 nodes and 19,495 links, the RP graph is composed of 8560 nodes and 11,782 links and the MT graph is composed of 11,782 nodes and 60,506 links. Note that Pearson coefficient between the stochastic matrices rises to $0.1776,0.6783$ and 0.4286 for RT/RP, RT/MT et $\mathrm{RP} / \mathrm{MT}$, respectively. Thus, the retweet and mention relationships are clearly correlated which may cause some problems for the proposed method, as we shall see, since it is well known that least squares method is sensitive to that. Since the data is related to the world cup, the most used hashtags of bipartite users-hashtags graph UH are those referring to the 32 countries involved in the final phase as well as those referring directly to the event (\#WorldCup2014, \#Brazil, \#Brasil2014, \#CM2014, etc.). The semi-finalists have the greatest in-strength (the in-strength of the node $j$ is $s_{j}^{i n}=\sum_{i} w_{i j}$ is the sum of inward link weights).

\section{Results}

We apply the proposed method to find if the hashtags posted by users (UH) can be explained by other relations (RT, RP, MT and their combinations). For instance, given a user $u$, explaining UH by RT-UH and MT-RP-UH means that the hashtags posted by $u$ are, to some extent, a combination of those posted by the users retweeted by $u$ and those posted by the users who received a response from users mentioned by $u$. In other words, we try to understand if, in the case of the football World Cup 2014, the probability that 
Table 1 Coefficients and $p$-values for linear regressions whose regressors correspond to meta-paths of length 2 to explain the user-hasthag distribution (UH). Model AO corresponds to the null model: no predictor but one intercept that is the average of the explained variable

\begin{tabular}{lllll}
\hline Mod. & Meta-Paths & Coef. & $\boldsymbol{p}$-values & $\boldsymbol{r}^{2}$ \\
\hline A0 & Average : 1.8704e-05 & & & 0 \\
A1 & RT-UH & 0.6273 & - & 0.3594 \\
B1 & 0.4291 & - & 0.2289 \\
C1 & RP-UH & 1.0289 & - & 0.4606 \\
A2 & MT-UH & 0.5795 & 0.0062 & 0.6116 \\
& RT-UH & 0.3957 & 0.0105 & \\
B2 & RP-UH & -0.3578 & 0.0612 & 0.5943 \\
& RT-UH & 1.4534 & 0.0087 & 0.6111 \\
C2 & MT-UH & 0.0051 & 0.0138 & \\
& RP-UH & 0.9391 & 0.0057 & 0.6818 \\
A3 & MT-UH & -0.1283 & 0.0791 & \\
& RT-UH & 0.0791 & 0.0113 & \\
& RP-UH & 1.1466 & 0.0111 & \\
\hline
\end{tabular}

users post hashtags can be explained by the relations these users have with other users and the probability that these latter have to post these hashtags.

\section{Meta-paths of length 2}

We test linear regression models with all the possible combinations of meta-paths of length 2 (see Table 1). This test allows a first glimpse of the contribution of the simplest predictors. First, the more the predictors, the better the value of $r^{2}$. It, thus, could be tempting to consider them all. Nevertheless, it does not mean that all predictors are significant. Indeed, the analysis of the coefficients and $p$-values makes it possible to realize the correlation of some variables. In models B2 and A3, the RT-UH and MT-UH meta-paths are both present. However, the $p$-value associated with RT-UH is greater than 0.05 which states that we accept the null hypothesis for this predictor. This could be a consequence of the correlation between RT-UH and MT-UH.

In summary and as it can be seen in Table 1, the best model according to the $r^{2}$ and the $p$-values with threshold $\alpha=0.05$ would be the model A2 whose predictors are RT-UH and RP-UH. The gain in the $r^{2}$ with respect to any other model with 1 regressor (and so simpler model) is worth it, i.e., important $r^{2}$ improvement and not really more complexity added. This means that, for a given user, the hashtags they post can be explained by the hashtags posted by the users they retweet with a contribution of 0.5795 and the users they reply to with a contribution of 0.3957 . This model accounts for $61.16 \%$ of the variance.

\section{Importance of meta-path length}

This subsection looks at the length of the meta-paths for a given link type. More specifically, we compute, for each link type, the $r^{2}$ score when the only predictor is associated with a random walk of length $l=1, \ldots, 10$ repeating the same link type. For instance, for $l=2$ and the retweet action, the predictor will be RT-RT-UH representing the hashtags posted by people who are retweeted by people who are themselves retweeted. Intuitively, the importance of a meta-path decreases with its length $(=l+1)$ since considering longer meta-paths means considering more extended neighborhoods, hence the information is 

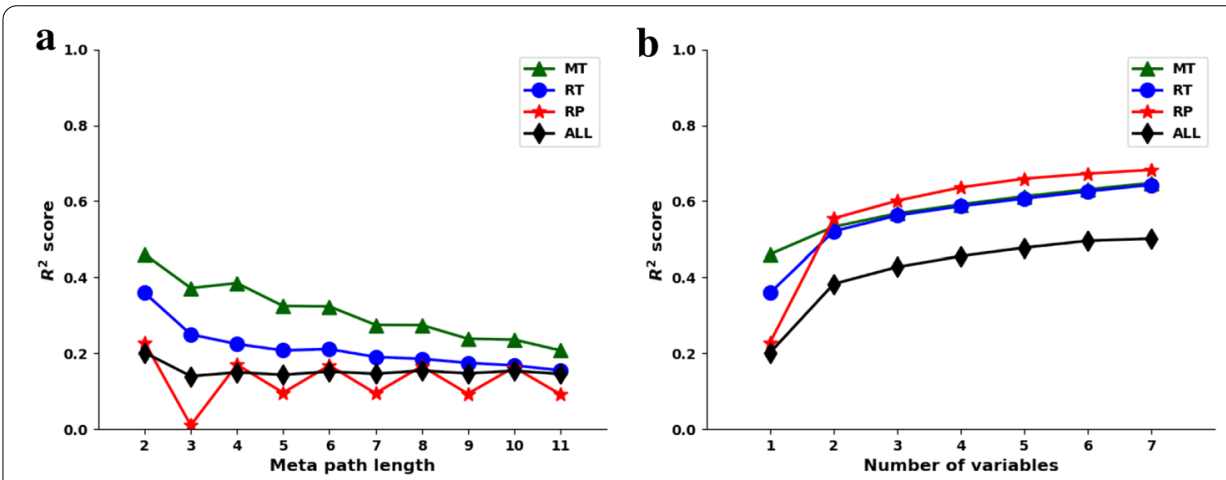

Fig. 3 a Linear regression $r^{2}$ scores with one predictor associated with a meta-path whose length varies between 2 and 11. $\mathbf{b}$ Linear regression $r^{2}$ scores according to the number of meta-paths of the same link type (see main text for explanation)

more diffused. By way of illustration, the walker can attain a lot of nodes with some of them really far from the starting node. This is corroborated in Fig. 3a where we can see a tendency to decrease with respect to the meta-path length. Each link type brings a different quantity of information and the MT type is the more informative for our purpose.

Plus, this analysis exposes a characteristic of the reply dynamics: most of the time, the replies involved only two people [47]. This is reflected through the oscillations of the reply scores in Fig. 3a. The scores associated with odd length random walks are low since the walker is forbidden to return to the initial node on the penultimate step of the walk (Fig. 4).

For simpler hypothesis, we also draw in black the $r^{2}$ scores when we do not differentiate the link types (ALL), i.e., all the link weights between nodes are aggregated. This score is below the average score of the three specific link types. One can see that just taking the mention or retweet type is more informative than the aggregation which reinforces the relevance of differentiating the link types.

Fig. $3 \mathrm{~b}$ shows $r^{2}$ scores when we combine variables of different lengths related to the same link type in the model. Actually, the $r^{2}$ associated with $n$ number of variables is related to the model whose predictors are all meta-paths of length smaller or equals to $n+1$ and whose the steps except the last are in the same type of links. For instance, for $n=3$ variables, the predictors are RT-UH, RT-RT-UH and RT-RT-RT-UH (for the RT case). Again, the more the variables, the better the score. Also, the increase is not linear; the best improvement happens when we combine length-1 and length-2 variables which indicates the need to consider them together. We can also observe that scores given by the RT and MT types are really similar when considering more than two variables while there is a clear difference in the $r^{2}$ score for single variable. It means that their respective combinations have the same result in term of $r^{2}$ although the underlying semantics are different. Once again, the $r^{2}$ score for the aggregation is shown and is far below the other scores. This indicates the importance of distinguishing the types of links.

Since it is often desirable to keep a model simple both in term of interpretability and computation time, there is a trade-off between the highest possible $r^{2}$ and the cost to attain it. The tests here performed tend to show that considering too long as well as too many meta-paths is not necessarily useful in our case. Indeed, the gain in the $r^{2}$ is not worth it considering the complexity it brings. This is in accordance with work dealing with 


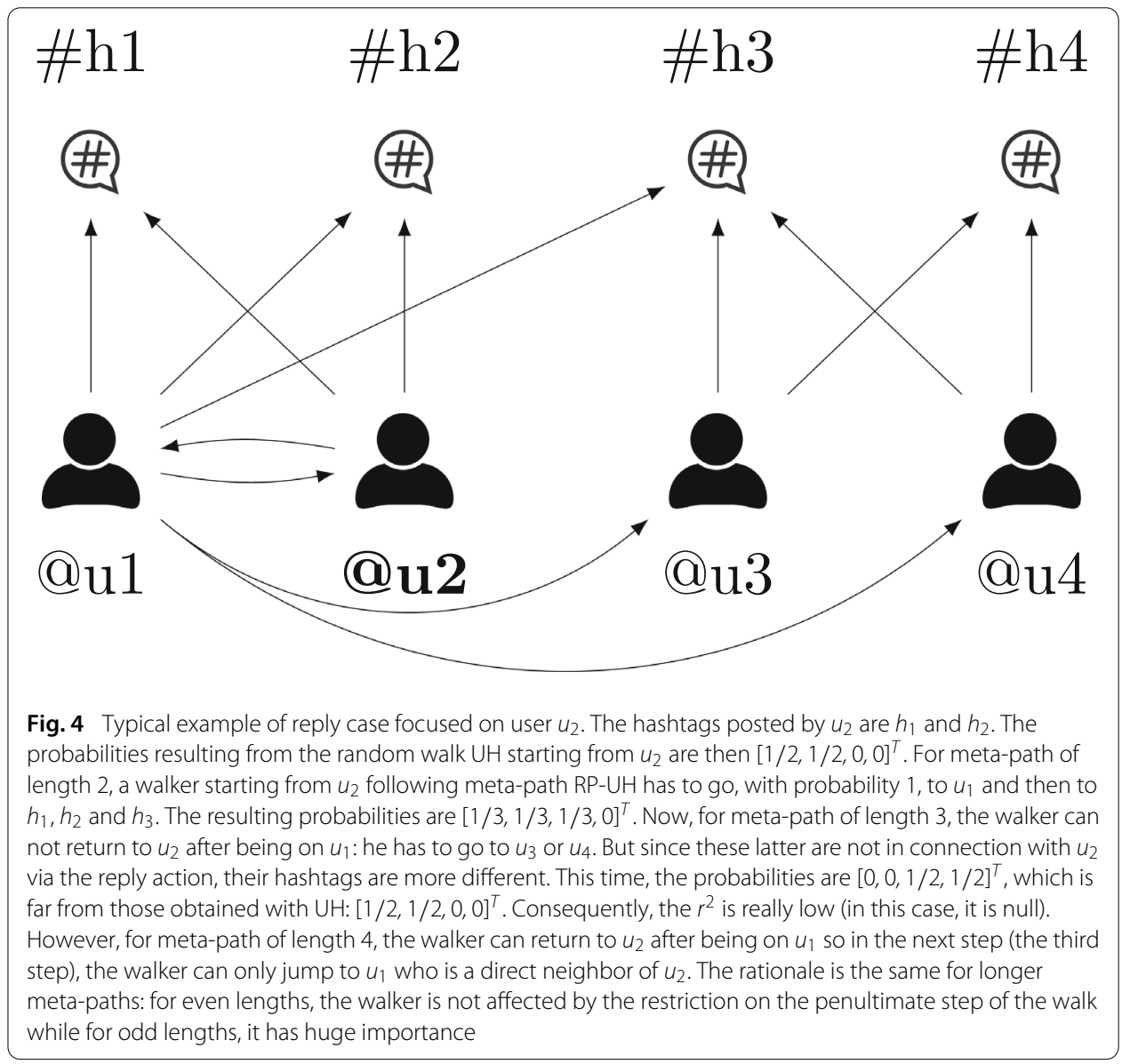

other purposes as node similarity or clustering: meta-path with relatively short length is sufficient to evaluate similarity scores, and a longer meta-path may even deteriorate the quality $[28,31]$.

\section{Forward linear regression for data description}

We apply the proposed algorithm on the entire dataset with a threshold $\alpha=0.05$ for $p$-values. As a reminder, the procedure stops when it is no longer possible to improve the $r^{2}$ by adding significant regressors. Since the length of meta-paths is unbounded, the set of possible meta-paths is infinite. Here, the $k$ potential predictors are those of length less than or equal to 4 . This is motivated by the test performed in the previous subsection. In addition, the semantics of longer paths are less clear than shorter paths.

Results are reported in Table 2. The final model thus obtained contains five predictors related to meta-paths whose length are no longer than 3 and no intercept. This regression model accounts for $71.29 \%$ of the variance. To comfort the goodness of fit of the model, we plot in Fig. 5 the density plot in log-log scale of the predicted probabilities versus the observed ones in the data. The green line represents the ideal case where predicted probabilities match observed ones. Most of the data points fall to this line which reinforces the use of a linear model.

The best improvement with respect to $r^{2}$ comes with the addition of the second variable (see Mod. 2 of Table 2). The model with two predictors is actually a local extremum since 
Table 2 Results of the forward stepwise linear regression

\begin{tabular}{lllll}
\hline Mod. & Meta-paths & Coef. & $\boldsymbol{p}$-values & $\boldsymbol{r}^{\mathbf{2}}$ \\
\hline 0 & Average: $1.8704 \mathrm{e}-05$ & & & 0 \\
1 & MT-UH & 1.0289 & - & 0.4606 \\
2 & MT-UH & 0.9391 & 0.0057 & 0.6112 \\
& RP-UH & 0.0052 & 0.0137 & 0.6682 \\
& MT-UH & 0.8464 & 0.0062 & \\
& RP-UH & 0.0335 & 0.0124 & 0.6947 \\
4 & RT-RP-UH & 0.1077 & 0.0138 & \\
& MT-UH & 0.8114 & 0.0063 & \\
& RP-UH & 0.0362 & 0.0109 & \\
& RT-RP-UH & 0.0766 & 0.0142 & \\
5 & RP-MT-UH & 0.0676 & 0.0143 & \\
& MT-UH & 0.1974 & 0.0094 & \\
& RP-UH & 0.5556 & 0.0146 & \\
& RT-RP-UH & 0.0650 & 0.0125 & \\
& RP-MT-UH & 0.1591 & 0.0160 & \\
& MT-RT-UH & 0.0074 & 0.0124 & \\
\end{tabular}

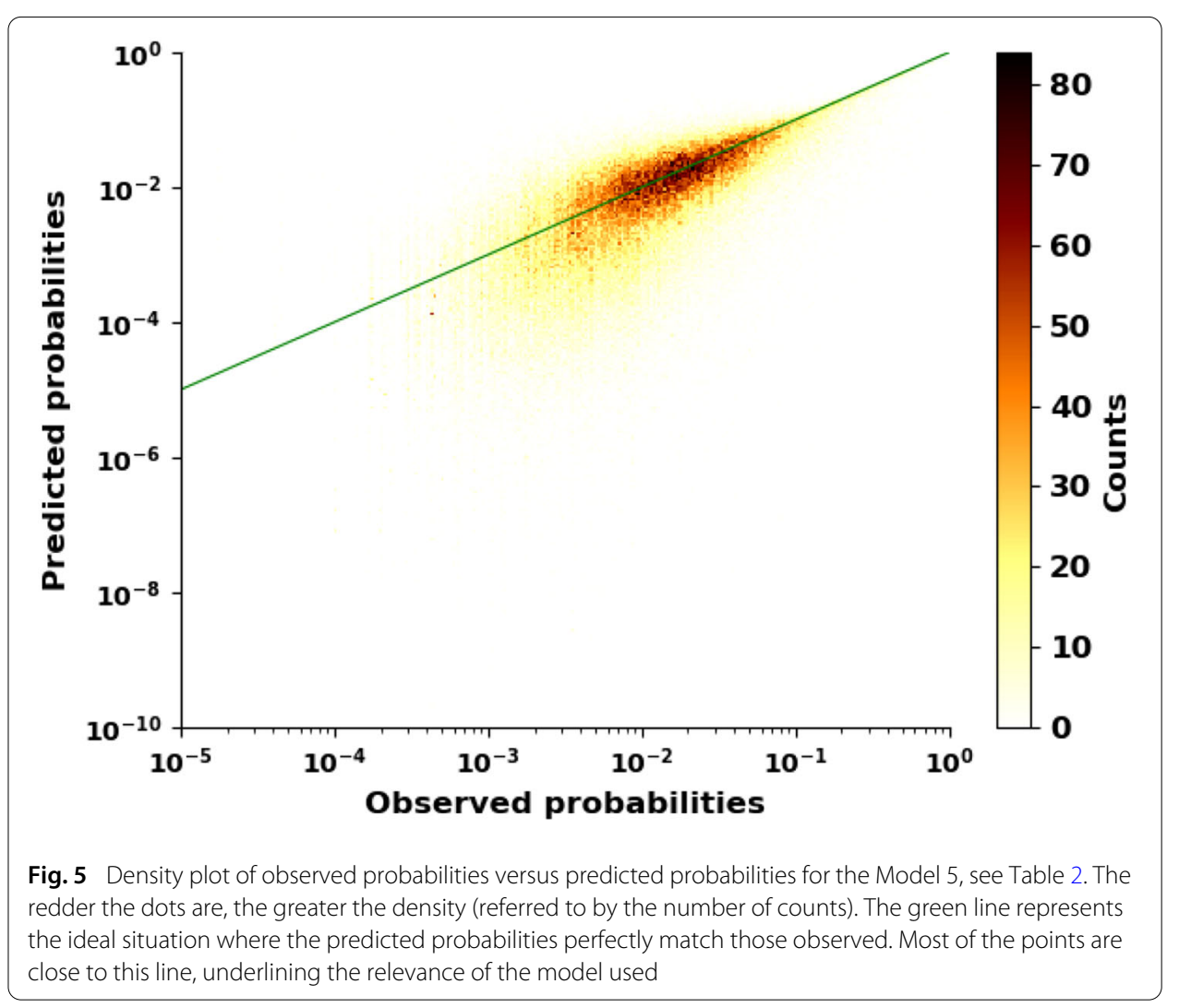

the model with the best $r^{2}$ is the one with RT-UH and RP-UH predictors (see Table 1). Although the difference is tenuous, this allows to point two weaknesses of the method: there is no guarantee of finding the best model and the order of the variable selection is important. Note that the first two variables are part of the most direct relationships (metapaths of length 2) which is intuitive: the direct neighborhood of a user shares common topics of interest with them. The last meta-path included in the model (Mod. 5) provokes 
Table 3 Summary of the final models for the three similarity measures considered: PCRW, PC and AvgSim. For each similarity measure, we indicate the values of the coefficient associated with the meta-path. The number before the coefficient indicates the order in which meta-paths are included in the final model

\begin{tabular}{llll}
\hline Meta-Paths & PCRW & PC & Avgsim \\
\hline RT-UH & & & 5) 0.1629 \\
RP-UH & 2) 0.5556 & 2) 0.2534 & \\
MT-UH & 1) 0.1974 & 1) 0.5550 & 1) 0.5567 \\
RT-RP-UH & 3) 0.0650 & 3) 0.0289 & 3) 0.0380 \\
RT-MT-UH & & 5) 0.0763 & \\
RP-RP-UH & & 2) 0.3840 \\
RP-MT-UH & 4) 0.1591 & 4) 0.0216 & \\
MT-RT-UH & 5) 0.0074 & & 6) 0.1070 \\
MT-RP-UH & & 0.6778 & 4) 0.1154 \\
$r^{2}$ & 0.7129 & & 0.7649 \\
\hline
\end{tabular}

an important change in the other coefficients. After exploration, this is due to the presence of outliers in this predictor, i.e., observations that differ greatly from the trend expressed by the other observations. It is well known that ordinary least squares method is sensitive to that. Indeed, after a quite rough identification of these outliers ${ }^{2}$ and their imputation by the mean of the values of the predictor in question, the meta-path MT-RT-UH is no longer part of the model and the algorithm stops after the fourth iteration.

We apply the same procedure with other similarity measures, as explained in "OtherMeasures" section. As one can see in Table 3, results are different. However, the meta-path MT-UH is always the first to enter into the model. PCRW and PC are the most similar in term of the presence of meta-paths, a reason could be the fact they both just consider the "one way random walk", unlike AvgSim that computes a "round trip random walk". Finally it seems that for this dataset, the AvgSim similarity measure is more suited.

\section{Forward linear regression for data recovery}

We validate the method by performing a task aiming to recover the weights of missing links. In other words, this part tries to answer the following question: is it possible to know, in a quantitative way, the way some people post some hashtags knowing the way other people do?

We perform Monte Carlo cross-validation with $80 \%$ of the users as the training set and obtain the vector $\beta$ for them. Then, we use it on the testing set, i.e., the remaining $20 \%$ and compute the $r^{2}$ associated with each model. We proceed to ten splits, i.e., we create ten training sets. The final models do not include the same variables as before. Not surprisingly, it depends on the $80 \%$ selected. The number of predictors is five or six. Nevertheless, whatever the training set, the meta-path MT-UH is always the first predictor to be selected. After, there is no more consensus on the second regressor but the RP-UH and RT-RP-UH always compete for the second place. Again, it is not surprising to obtain the RP-UH meta-path since, for a user, it is related to one of the closest neighbors with respect to our graph construction and very weakly correlated to the MT-UH meta-path already present in the model. Although the best $r^{2}$ scores of the final models reach, on

${ }^{2}$ Data points outside the outer fences, i.e., outside the interval [Q1 - (3"IQR), Q3 + (3"IQR)] with Q1, Q3 and IQR=Q3Q1 the lower, upper quartile and interquartile range, respectively. 


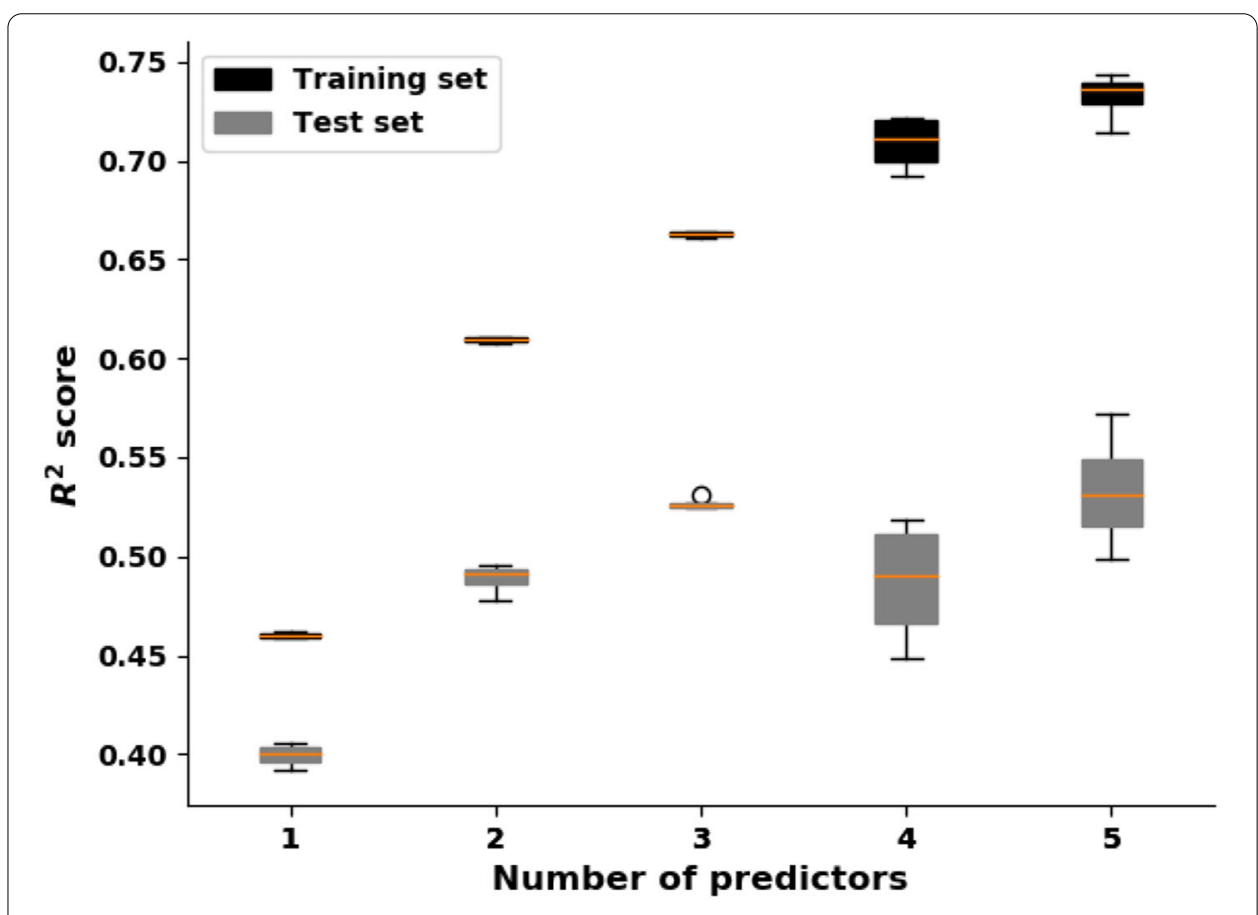

Fig. 6 Boxplot of the $r^{2}$ scores of training sets (black) and test sets (gray). The training set scores increase with the number of predictors in the model while for the testing set, the scores seem to reach a threshold

average, 0.7 for the training sets, we only get, on average, a best score of 0.5 for the test sets (Fig. 6). The method seems to reach a limit. One also observes that even if a model better fits the training set, it does not mean that it will give the best recovery. Indeed, it is sometimes better to consider a model with fewer regressors, and so a lower $r^{2}$ for training set, to better recover.

Since $r_{P C}^{2}<r_{P C R W}^{2}$ for the description task (see Table 3), we do not use it for the recovery task. However, we apply the AvgSim for which we obtain, on average, $r_{r e c, A S}^{2}=0.55$, still a little bit better than with PCRW.

\section{Bibliographic data}

Bibliographic networks are also good examples of heterogeneous information networks since they contain multiple types of nodes and links. We here focus on scientific publications.

\section{Dataset description and setup}

Figure 7 illustrates an example of such networks where one can distinguish four types of nodes that is authors, papers, venues and topics; and four types of links (eight when we differentiate a type from its inverse) that is write, publish, cite and belong to.

The HIN analyzed in this article is constructed from DBLP publications [48]. The dataset contains 95,855 authors with 1,537,407 co-author relationships and 186,175 papers with $1,356,893$ citation relationships. The papers belong to nine distinct topics: Artificial Intelligence A.I., Computer Graphic: multimedia C.G., Computer Networks C.N., Database: Data Mining: Information Retrieval, Human Computer Interaction: Ubiquitous Comput- 


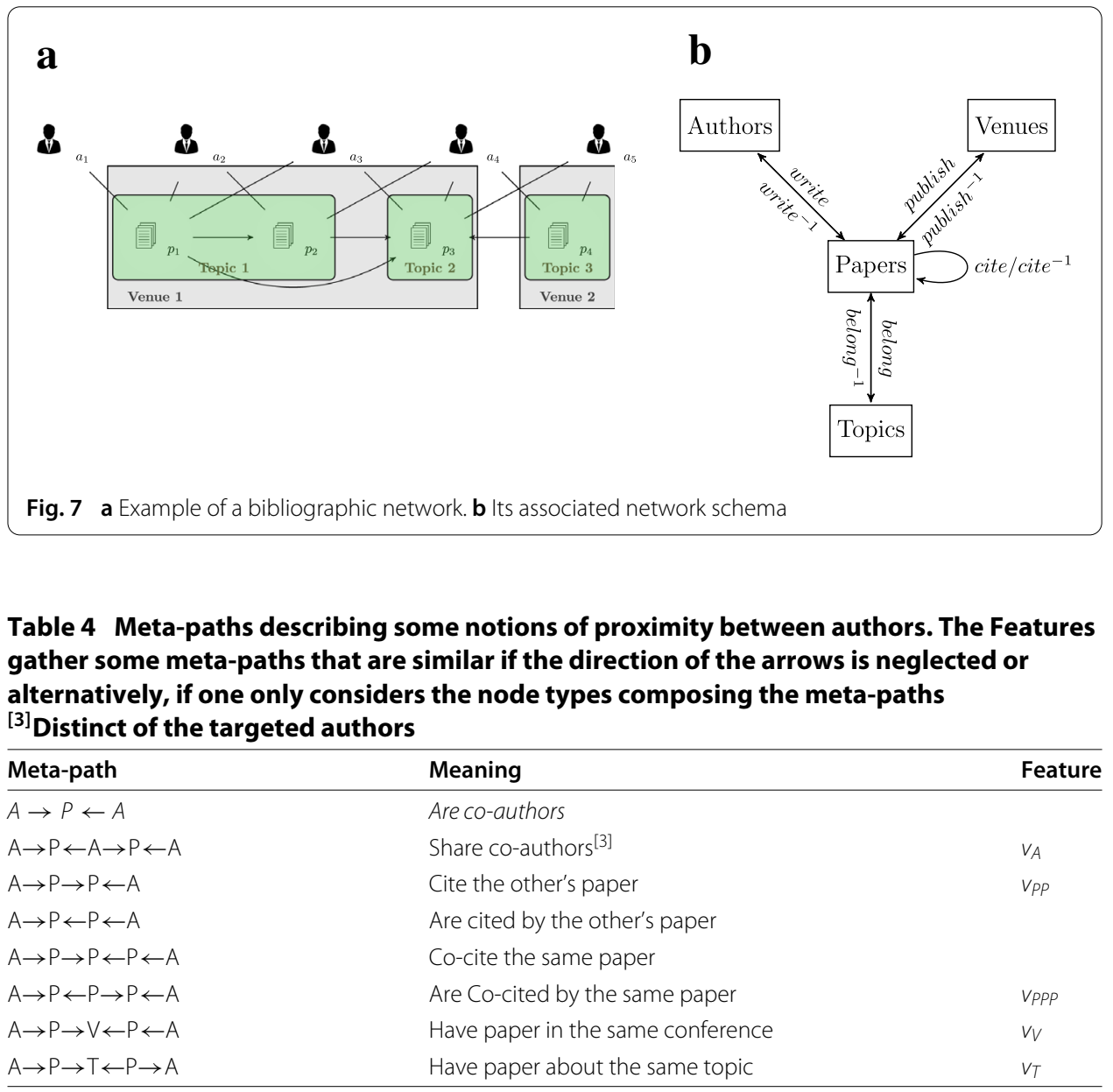

ing H.C.I., Information Security I.S., Interdisciplinary Studies I.St., Software Engineering S.E. and Theoretical Computer Science T.C.S.. These topics are represented in the 92 venues present in the dataset.

The presented method is used to find out if the co-author relationship $\mathrm{A} \rightarrow \mathrm{P} \leftarrow \mathrm{A}$ is correlated with other directly extractable relationships of the underlying graph. Table 4 shows the different meta-paths used in the models selected according to their semantics contrary to the previous experiment. Since there is only one directed type of links between two given types of nodes, we only mention the types of nodes to describe the meta-paths.

As mentioned, meta-paths are no longer determined by their length but selected by a more solid prior knowledge about the data. Here are given some motivations about the selected meta-paths.

- $\mathrm{A} \rightarrow \mathrm{P} \rightarrow \mathrm{A} \leftarrow \mathrm{P} \rightarrow \mathrm{A}$ means that two authors have written with a third common author. It represents a triangle when the AP-PA graph is projected onto A. This meta-path is the most "social";

- $\mathrm{A} \rightarrow \mathrm{P} \rightarrow \mathrm{P} \leftarrow \mathrm{A}$ and $\mathrm{A} \rightarrow \mathrm{P} \leftarrow \mathrm{P} \leftarrow \mathrm{A}$ state for the interest of a person (say $a$ ) for the work of another (say $b$ ). It could be meaningful to think that if $a$ is interested in $b$ 's work and cites it, $a$ is eager to communicate with $b$ and even to collaborate and to publish with her. The same holds if $a$ and $b$ exchange their role; 
- $\mathrm{A} \rightarrow \mathrm{P} \rightarrow \mathrm{P} \leftarrow \mathrm{P} \leftarrow \mathrm{A}$ means that two authors cite the same paper and are, thus, inspired by the same ideas. This could be a good reason for a co-author relation;

- $\mathrm{A} \rightarrow \mathrm{P} \leftarrow \mathrm{P} \rightarrow \mathrm{P} \leftarrow \mathrm{A}$ is quite different since it states that a third person (say $c$ ) cites the work of $a$ et $b$ but it does not mean that $a$ and $b$ work on the same thing. So, we expect this meta-path to be less significant than the previous one, albeit the structure is fairly close;

- $\mathrm{A} \rightarrow \mathrm{P} \rightarrow \mathrm{V} \leftarrow \mathrm{P} \leftarrow \mathrm{A}$ and $\mathrm{A} \rightarrow \mathrm{P} \rightarrow \mathrm{T} \leftarrow \mathrm{P} \leftarrow \mathrm{A}$ mean that $a$ 's paper and $b$ 's paper are in the same venue or belong to the same topic, respectively. Even if some venues can gather a lot of people, being accepted in the same venue might trigger collaborations. Plus, working on the same topic can also be a source of collaboration.

Starting from the data, we construct four matrices associated with four bipartite graphs. In particular, $A P$ where $A P_{a p}$ equals 1 when authors $a$ writes paper $p, 0$ otherwise. $P P$ where $P P_{p q}$ equals 1 when paper $p$ cites paper $q, 0$ otherwise. $P V$ where $P V_{p v}$ equals 1 when paper $p$ is published/presented in conference/venue $v, 0$ otherwise. $P T$ where $P T_{p t}$ equals 1 when paper $p$ belongs to topic $t, 0$ otherwise. These matrices are binary but it does not imply the co-author matrix $(A A)$ is binary too. To compute the proposed variables/meta-paths, matrices are transformed into row-stochastic matrices, i.e., normalized such that the sum of each line equals 1 . In this setting, we can consider these matrices as transition matrices and perform random walks on it. For Fig. 7a, we have the following matrices:

$$
\begin{aligned}
& A P=\left[\begin{array}{ccccc}
1 & 0 & 0 & 0 & 0 \\
1 / 2 & 1 / 2 & 0 & 0 & 0 \\
1 / 2 & 0 & 1 / 2 & 0 & 0 \\
0 & 1 / 3 & 1 / 3 & 1 / 3 & 0 \\
0 & 0 & 1 / 2 & 1 / 2 & 0 \\
0 & 0 & 0 & 0 & 1
\end{array}\right] \quad P V=\left[\begin{array}{ccc}
1 & 0 & 0 \\
1 & 0 & 0 \\
1 & 0 & 0 \\
0 & 1 & 0 \\
0 & 0 & 1
\end{array}\right] \\
& P P=\left[\begin{array}{ccccc}
0 & 1 / 2 & 1 / 2 & 0 & 0 \\
0 & 0 & 1 & 0 & 0 \\
0 & 0 & 0 & 0 & 1 \\
0 & 0 & 1 & 0 & 0 \\
0 & 0 & 0 & 0 & 1
\end{array}\right] \quad P T=\left[\begin{array}{cccc}
1 & 0 & 0 & 0 \\
1 & 0 & 0 & 0 \\
0 & 1 & 0 & 0 \\
0 & 0 & 1 & 0 \\
0 & 0 & 0 & 1
\end{array}\right]
\end{aligned}
$$

where the red entries (last columns and rows of each matrix) are related to the so-called hole nodes (see Sec. ). Remark that paper $p_{3}$ points to the hole node in the PP graph since it does not cite any paper.

Furthermore, note that for meta-paths of the form $\mathrm{A} \rightarrow \mathrm{P} \rightarrow$ "node type" $\leftarrow \mathrm{P} \leftarrow \mathrm{A}$ with "node type" in $\{\mathrm{P}, \mathrm{V}, \mathrm{T}\}$, the walker is forbidden to return to the same paper in his second and fourth step. It prevents us from using what we are looking for. For instance, in Fig. 7, a walker constrained by the $\mathrm{A} \rightarrow \mathrm{P} \rightarrow \mathrm{A} \leftarrow \mathrm{P} \leftarrow \mathrm{A}$ meta-path and having traveled through the path $a_{1} \rightarrow p_{1} \rightarrow a_{3}$ cannot return on $p_{1}$ at their next step but has to go to $p_{3}$.

\section{Results}

As said, the aim of this experiment is to express the distribution of co-author relationship of all the authors in the dataset by a combination of other distributions. The results are once again divided into explanatory and recovery tasks. 
Table 5 Results of the linear model for all selected meta-paths

\begin{tabular}{lll}
\hline Meta-Path & Coefficient & $\boldsymbol{p}$-value \\
\hline$A \rightarrow P \leftarrow A \rightarrow P \leftarrow A$ & 1.2507 & 0.0038 \\
$A \rightarrow P \rightarrow P \leftarrow A$ & 0.9237 & 0.0099 \\
$A \rightarrow P \leftarrow P \leftarrow A$ & - & - \\
$A \rightarrow P \rightarrow P \leftarrow P \leftarrow A$ & 0.2813 & 0.0395 \\
$A \rightarrow P \leftarrow P \rightarrow P \leftarrow A$ & - & - \\
$A \rightarrow P \rightarrow V \leftarrow P \leftarrow A$ & 0.1539 & 0.0099 \\
$A \rightarrow P \rightarrow T \leftarrow P \leftarrow A$ & - & - \\
$r^{2}$ & 0.6661 & \\
\hline
\end{tabular}

Table 6 Results of the linear model for meta-paths aggregated into features

\begin{tabular}{lll}
\hline Feature & Coefficient & $\boldsymbol{p}$-value \\
\hline$V_{A}$ & 1.2133 & 0.0028 \\
$V_{P P}$ & 1.8549 & 0.0034 \\
$V_{P P P}$ & - & - \\
$V_{V}$ & - & - \\
$V_{T}$ & - & - \\
$r^{2}$ & 0.5997 & \\
\hline
\end{tabular}

\section{Forward liner regression for data description}

Two tests are performed: first, we consider all the presented meta-paths as regressors (Table 5) and second, we aggregate some meta-paths a.k.a. features (see third column of Table 4) and utilize them into the algorithm (Table 6). We propose this aggregation because if the direction of the arrows is neglected, the meta-paths composing a feature are the same. In other words, the sequence of the node types is the same. The aim is to quantify the quality loss (if any) of the prediction when aggregating meta-paths into features.

Meta-paths as regressors Only three meta-paths are retained into the final model. This latter is able to explain $66,61 \%$ of the variance in the dependent variable from the independent variables. According to this model, the most significant meta-paths to explain the co-author relationship are related to the way authors share the same co-authors (some kind of transitivity ${ }^{3}$ ), cite and co-cite, plus the venues in which papers are published/presented. Meta-path related to "topic" is not included in the model.

Features as regressors When meta-paths are aggregated into features, those related to citing the same paper and the venues are not included in the model (see Table 6). For the first one, it could be explained by the fact that only one meta-path $(\mathrm{A} \rightarrow \mathrm{P} \rightarrow \mathrm{P} \leftarrow \mathrm{P} \leftarrow \mathrm{A})$ among two is imported in the first test. ${ }^{4}$

No immediate reason is given for the absence of $v_{V}$ variable. Plus, this second model only accounts for $59.97 \%$ of the variance: each meta-path brings its own meaning and even if some of them seem close to each other, wanting to aggregate them is not beneficial for our purpose. Actually, we have already mentioned a fundamental difference between variables of $v_{P P P}$. As in the previous case, feature related to "topic" is not significant for the specific objective when other variables (see Table 4) are considered in the forward linear regression.

\footnotetext{
${ }^{3}$ Transitivity of the authors-authors graph equals 0.6948 .

${ }^{4}$ Same remark can be made for the $v_{P P}$ meta-paths and yet, $v_{P P}$ is part of the model.
} 
Table 7 Results of the different topics

\begin{tabular}{lllllll}
\hline Topic & \#auth. & \#pap. & \#ven. & $\boldsymbol{r}^{\mathbf{2}}$ & $\boldsymbol{r}_{\boldsymbol{P C}}^{\mathbf{2}}$ & $r_{\boldsymbol{A S}}^{\mathbf{2}}$ \\
\hline A.I. & 41538 & 65927 & 23 & 0.5914 & 0.5010 & 0.5372 \\
C.G.M. & 25989 & 18877 & 13 & 0.6358 & 0.5313 & 0.5226 \\
C.N. & 22374 & 30212 & 9 & 0.6321 & 0.5152 & 0.5730 \\
Database & 5865 & 9294 & 7 & 0.7349 & 0.6954 & 0.7570 \\
H.C.I. & 4660 & 10666 & 5 & 0.7723 & 0.7242 & 0.7830 \\
I.S. & 5298 & 6943 & 6 & 0.7211 & 0.6707 & 0.7919 \\
I.St. & 46111 & 2614 & 11 & 0.7838 & 0.7037 & 0.6759 \\
S.E. & 8147 & 20506 & 8 & 0.7222 & 0.6465 & 0.6509 \\
T.C.S. & 10824 & 21136 & 11 & 0.5796 & 0.4409 & 0.4266 \\
\hline
\end{tabular}

No perfect answer exists to determine which predictors are the best between metapaths and features since there is always a trade-off between complexity and efficiency. Nevertheless, results obtained with meta-paths are satisfactory and in addition, it is easier to associate a semantics to these latter.

Topics under scrutiny The small number of considered topics, compared with the number of papers, could partly explain why the topic meta-path is not taken into account. Indeed, only one topic is assigned to each paper so the meta-path $\mathrm{P} \rightarrow \mathrm{T} \rightarrow \mathrm{P}$ generate a dense "paper-paper matrix" 5 and when computing the matrix product $\mathrm{A} \rightarrow \mathrm{P} \rightarrow \mathrm{T} \leftarrow \mathrm{P} \leftarrow \mathrm{A}$, any relevant information is somewhat lost.

Thus, we think the meta-path $\mathrm{A} \rightarrow \mathrm{P} \rightarrow \mathrm{T} \leftarrow \mathrm{P} \leftarrow \mathrm{A}$ brings a too diffuse information. However, the idea of considering topics is not meaningless since an author interested in a topic is often interested for a while and therefore, has the time to collaborate with other people, who are themselves interested in the same subject. Authors writing about a same topic might partly be co-authors.

So, we split the data into nine subsets, each one related to one topic, and apply the method with the six meta-paths cited above, i.e., all except $\mathrm{A} \rightarrow \mathrm{P} \rightarrow \mathrm{T} \leftarrow \mathrm{P} \leftarrow \mathrm{A}$ (see Remark 2). Results are reported in Table 7. On average, we have a better descriptive model than before: $\left\langle r^{2}\right\rangle=0.6970$ (and $\sigma=0.0710$ ). This could mean that inside some topics, there are some patterns more homogeneous or frequent and we are more capable of explaining them. However, for Artificial Intelligence and Theoretical Computer Science, it is harder to find a model that matches the data.

For comparison, we also report the $r^{2}$ obtained with PathCount and AvgSim. As one can see, PathCount is always surpassed by the other two; and these latter compete depending on the topic.

The final models' coefficient values for the different topics are reported in Fig. 8. For the sake of brevity, we only report the coefficients for PCRW. Meta-path $\mathrm{A} \rightarrow \mathrm{P} \rightarrow \mathrm{A} \leftarrow \mathrm{P} \leftarrow \mathrm{A}$ is selected by each topic: sharing the same co-author is the most useful to explain co-author relationship in a given topic. Meta-path $\mathrm{A} \rightarrow \mathrm{P} \rightarrow \mathrm{V} \leftarrow \mathrm{P} \leftarrow \mathrm{A}$ is important for 7 topics out of 9. Only Computer Networks and Theoretical Computer Science do not take it into account. Note that only Theoretical Computer Science includes $\mathrm{A} \rightarrow \mathrm{P} \leftarrow \mathrm{P} \rightarrow \mathrm{P} \leftarrow \mathrm{A}$ in its

${ }^{5}$ The same comment could be made for meta-path $\mathrm{P} \rightarrow \mathrm{V} \rightarrow \mathrm{P}$ since the number of venues is also limited - although to a lesser extent since a topic encompasses several venues. The number of non-zero entries of the matrix APTPA (not really the same as PTP but the final result is encompassed in APTPA) equals 6,515,232; while for APVPA, this number raises to $3,940,634$, which is still 1.6 times lower. 


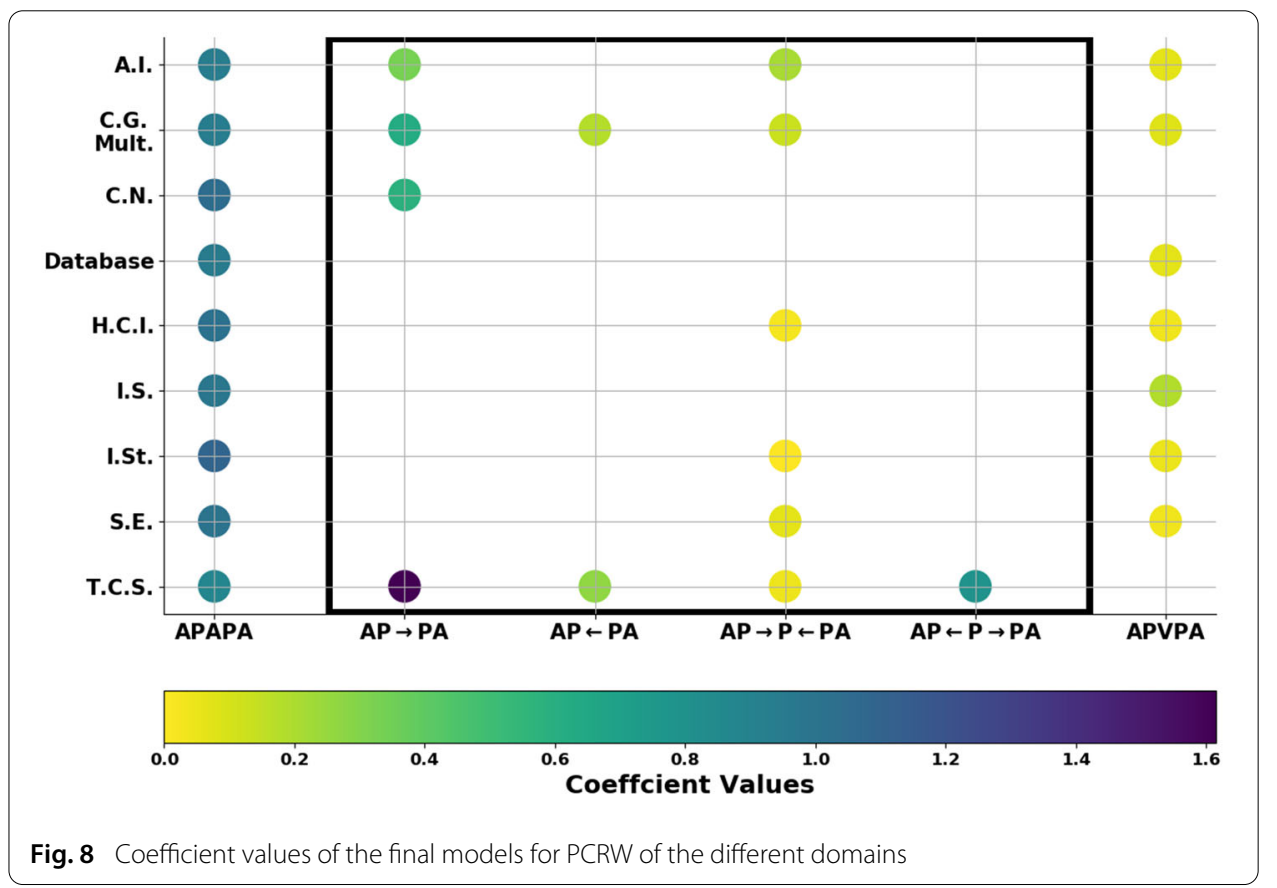

final model. This topic is also the only one for which $\mathrm{A} \rightarrow \mathrm{P} \rightarrow \mathrm{A} \leftarrow \mathrm{P} \leftarrow \mathrm{A}$ has not the greatest coefficient, it is surpassed by $\mathrm{A} \rightarrow \mathrm{P} \rightarrow \mathrm{P} \leftarrow \mathrm{A}$ and closely followed by $\mathrm{A} \rightarrow \mathrm{P} \rightarrow \mathrm{P} \leftarrow \mathrm{P} \leftarrow \mathrm{A}$. The paper relations seem highly important for this domain.

As one can see, meta-paths in the rectangle are less present than the other two. Indeed, many possible configurations hide behind a single meta-path. An interesting point, thus, lies in the self-citations. For instance, let's take a look at $\mathrm{A} \rightarrow \mathrm{P} \rightarrow \mathrm{P} \leftarrow \mathrm{A}$ and the underlying configurations where white (black) circles stand for authors (papers).

\begin{tabular}{|c|c|c|c|c|}
\hline & ?: & 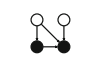 & $\because$ & 9 \\
\hline A. I. & 1,242 & 117 & 120 & 41 \\
\hline Comp. Graph. Mult. & 10,632 & 731 & 863 & 301 \\
\hline Comp. Net. & 28,732 & 1,050 & 1,011 & 553 \\
\hline Database & 4,333 & 162 & 198 & 90 \\
\hline Hum. Con & 2,582 & 181 & 176 & 66 \\
\hline Info. & 900 & 57 & 81 & 31 \\
\hline & 157 & 1 & 2 & 0 \\
\hline Softr & 2,097 & 163 & 174 & 61 \\
\hline Th. Comp. Sci. & 839 & 65 & 59 & 24 \\
\hline
\end{tabular}

It is clear that $\mathrm{A} \rightarrow \mathrm{P} \rightarrow \mathrm{P} \leftarrow \mathrm{A}$ might encompass $\mathrm{A} \rightarrow \mathrm{P} \leftarrow \mathrm{A}$; for the first, it takes a third paper to be co-authors whilst for the other, they are already. So, a look at the sub-graphs and then motifs (i.e., significantly overrepresented sub-graphs) would be informative. However, this is not the scope of this work and it is left for future investigations.

\section{Forward linear regression for data recovery}

We are now interested in the recovery of link weights between authors. Average results Monte Carlo cross-validations are reported in Table 8. All $p$-values associated with the regressors are below the fixed threshold $\alpha=0.05$. 
Table 8 Results of the recovery task for the general case (all topics) and per topic. PCRW and AvgSim are used as similarity measures

\begin{tabular}{lll}
\hline Topics & $\left\langle\boldsymbol{r}_{\text {test } \boldsymbol{P} \text { PRW }}^{2}\right\rangle$ & $\left\langle\boldsymbol{r}_{\text {test } \boldsymbol{A S S}}^{\mathbf{2}}\right\rangle$ \\
\hline All topics & 0.5508 & 0.5742 \\
A.l. & 0.4994 & 0.4290 \\
Comp. Graph. Mult. & 0.5133 & 0.4743 \\
Comp. Net. & 0.5322 & 0.4911 \\
Database & 0.7258 & 0.7057 \\
Hum. Comp. Inter. & 0.7338 & 0.7584 \\
Info. Sec. & 0.6509 & 0.7367 \\
Interdisc. Std. & 0.7440 & 0.7688 \\
Software Eng. & 0.6450 & 0.6130 \\
T.C.S. & 0.3557 & 0.3487 \\
\hline
\end{tabular}

Since PathCount is always worst than the other two for descriptive purpose (Table 7), we only compute the recovery scores for PCRW and AvgSim.

For Database, Human Computer Interaction and Interdisciplinary Studies, the recovery is somehow achievable in the sense that the score of the test set is almost as good as for the training set. For the other domains, the quality loss is more significant, even for Information Security and Software Engineering which have a good $r^{2}$ for the training set. This time, $\left\langle r_{\text {test }, P C R W}^{2}\right\rangle$ for Database is greater than $\left\langle r_{\text {test }, A S}^{2}\right\rangle$ : this could come from the fact that AS overfits the data, while the inverse for Interdisciplinary Studies. Finally, note that for Theoretical Computer Sciences, the $r^{2}$ for recovery is really low and its true relevance can be somewhat even questioned (albeit the $p$-values are below 0.05). However, to be sure of its relevance, the results computed from our dataset are compared with a null hypothesis model that preserves some properties of the network topology (e.g., degree distributions) but randomly reshuffles the links among the nodes. The aim is to show that degree distributions only are not enough to generate such a correlation in the data and that this correlation arises from the particular data or at least, from more involved topological properties. Indeed, results for such null models are not significant (no regressor with $p$-value smaller than 0.12) and the average score $\left\langle r^{2}\right\rangle$ over 15 generations of null graphs are at most equal to 0.26 .

\section{Summary and discussion}

We have considered a linear combination of probability distributions resulting from pathconstrained random walks to explain, to some extent, a specific relation in heterogeneous information networks. This proposed method, highlighting the semantics present in the graph, allows to express the weight of a link between two nodes knowing some other links in a graph. This could be useful for prediction or recommendation tasks. For instance, we can draw a parallel with collaborative filtering [37], where the idea is that people often get the best recommendations from someone with similar tastes to their own. Concretely, for a HIN composed of two nodes types: users $U$ and items I, this could be related to our method with the meta-path $\mathrm{U} \rightarrow \mathrm{I} \rightarrow \mathrm{U} \rightarrow \mathrm{I}$. Indeed, the subpart $\mathrm{U} \rightarrow \mathrm{I} \rightarrow \mathrm{U}$ links users who are interested in the same item while the last step: $\mathrm{U} \rightarrow \mathrm{I}$ presents a new item to be recommended to the first users.

In our opinion, an interest of the method presented here is the traceability of the models' construction. Indeed, it allows one to follow the steps of the algorithm, i.e., the order in 
which the meta-paths are integrated. The final model is, thus, clearly understandable and its interpretability facilitated. Further, if one wants to use this method for recovery tasks, one can only consider the first $n$ steps of the algorithm if, e.g., one judges the training model's quality at the $n^{\text {th }}$ step sufficient (compared to the improvement until the final not worthy/necessary, since one knows the final result on the training set).

In particular, we have shown by working on Twitter data, that the hashtags posted by a specific user is mainly related to those posted by her direct neighborhood, especially the mention and reply neighborhood. This method has also shown that the retweet relation is not really useful for our purpose. Then, we have shown the applicability of the method to bibliographic data to recover the co-author relationship. It has been found that (data separated into) some topics are more suited to our method and so, the functioning of co-authors seemed to differ from one topic to another. Looking closer to the meta-paths included in the models together with their semantics has allowed us to realize that some meta-paths might be encompassed in other. Considering motifs instead of meta-paths could be a solution, thus paving the way for future work. Three similarity measures have been tested in the procedure namely PathCount, Path-Constrained Random Walk and AvgSim. Whatever the dataset, PathCount is below the other two and AvgSim performs, on average, slightly better than PCRW.

Nevertheless, the main drawback of the method is its sensitivity to outliers. Hence, more robust least square alternatives could be envisaged such that Least Trimmed Squares or parametric alternatives. Furthermore when there is no prior knowledge about the data, as for the Twitter data experiment, we had to provide all the meta-paths whose length is no longer than four. Even if it has been motivated by previous tests, this threshold is clearly data related. Hence, it could be interesting to build a method able to find relevant metapaths by itself. Finally, data have been aggregated in time. Consequently, the chronology of the events is ignored. Since it is possible to extract the time stamp of tweets or to take into account the papers' publication date, a future work could be the integration of time by defining a random walk process on temporal graphs [49] or by counting the temporal or causal paths $[50,51]$ (plus normalization). The walker can, thus, only follow time-respecting paths which can perhaps improve the quality of the model.

\section{Acknowledgements}

Not applicable.

Authors' contributions

HLB and RLP analyzed the data and wrote the paper.

Funding

This work is funded in part by the European Commission H2020 FETPROACT 2016-2017 program under grant 732942.

Availability of data and materials

The DBLP dataset analyzed during the current study is available in the Citation Network Dataset repository, https://www. aminer.cn/citation. The Twitter dataset is available from the corresponding author on reasonable request.

Compliance with ethical standards

Competing interests

The authors declare that they have no competing interests.

Author details

'Sorbonne Université, CNRS, LIP6, 75005 Paris, France, ${ }^{2}$ Institut des systémes complexes de Paris Île-de-France, ISC-PIF, UPS, 3611 Paris, France.

Received: 16 March 2020 Accepted: 19 December 2020

Published online: 23 March 2021 


\section{References}

1. Newman M. Networks: an introduction. New York: Oxford University Press, Inc.; 2010.

2. Boccaletti S, Latora V, Moreno Y, Chavez M, Hwang DU. Complex networks: structure and dynamics. Phys Rep. 2006;424(4):175-308.

3. Barrat A, Barthélemy M, Pastor-Satorras R, Vespignani A. The architecture of complex weighted networks. Proc Natl Acad Sci. 2004;101(11):3747-52.

4. Antoniou IE, Tsompa ET. Statistical Analysis of Weighted Networks. Discrete Dynamics in Nature and Society. 2008;2008:Article ID 375452, 16 p.-Article ID 375452, 16 p. Available from: http://eudml.org/doc/129796.

5. Shi C, Li Y, Zhang J, Sun Y, Philip SY. A survey of heterogeneous information network analysis. IEEE Trans Knowledge Data Eng. 2016;29(1):17-37.

6. Cui Y, Zhang L, Wang Q, Chen P, Xie C. Heterogeneous Network Linkage-weight Based Link Prediction in Bipartite Graph for Personalized Recommendation. Procedia Computer Science. Promoting Business Analytics and Quantitative Management of Technology: 4th International Conference on Information Technology and Quantitative Management (ITQM 2016). 2016;91:953 - 958. http://www.sciencedirect.com/science/article/pii/S1877050916313126.

7. Li J, Zhang L, Meng F, Li F. Recommendation Algorithm based on Link Prediction and Domain Knowledge in Retail Transactions. Procedia Computer Science. 2014;31:875-881. 2nd International Conference on Information Technology and Quantitative Management, ITQM 2014. http://www.sciencedirect.com/science/article/pii/ S187705091400516X.

8. Guisheng Y, Wansi Y, Yuxin D. A new link prediction algorithm: node link strength algorithm. In: 2014 IEEE Symposium on Computer Applications and Communications; 2014; p. 5-9.

9. Symeonidis P, Tiakas E, Manolopoulos Y. Transitive Node Similarity for Link Prediction in Social Networks with Positive and Negative Links. In: Proceedings of the Fourth ACM Conference on Recommender Systems. RecSys '10. New York. ACM; 2010; p. 183-190.

10. Zhou K, Michalak TP, Waniek M, Rahwan T, Vorobeychik Y. Attacking similarity-based link prediction in social networks. In: Proceedings of the 18th International Conference on Autonomous Agents and MultiAgent Systems. AAMAS '19. Richland, SC: International Foundation for Autonomous Agents and Multiagent Systems; 2019; p. 305-313. http://dl. acm.org/citation.cfm?id=3306127.3331707.

11. Liao H, Zeng A, Zhang YC. Predicting missing links via correlation between nodes. Physica a: statistical mechanics and its applications. 2015;436:216 - 223. http://www.sciencedirect.com/science/article/pii/S0378437115004240.

12. Zhu X, Tian H, Cai S, Huang J, Zhou T. Predicting missing links via significant paths. EPL (Europhysics Letters) 2014;106(1):18008. https://doi.org/10.1209/0295-5075/106/18008.

13. Zhu X, Tian H, Cai S, Zhou T. Erratum: Predicting missing links via significant paths. EPL (Europhysics Letters). 2014; 108(4):49901. Available from: https://doi.org/10.1209/2F0295-5075/2F108/49901.

14. Lao N, Cohen WW. Relational retrieval using a combination of path-constrained random walks. Mach Learn. 2010;81(1):53-67.

15. Sun Y, Barber R, Gupta M, Aggarwal CC, Han J. Co-author relationship prediction in heterogeneous bibliographic networks. In: 2011 International Conference on Advances in Social Networks Analysis and Mining. IEEE, New York. 2011; p. 121-128.

16. Botterman HL, Lamarche-Perrin R. Combining path-constrained random walks to recover link weights in heterogeneous information networks. In: CompleNet 2019 - 10th Conference on Complex Networks. Springer Proceedings in Complexity. Tarragona, Spain: Springer; 2019; p. 97-109. https://hal.archives-ouvertes.fr/hal-02085410.

17. Lü L, Zhou T. Link prediction in complex networks: a survey. Physica A Stat Mech Appl. 2011;390(6):1150-70.

18. Martínez V, Berzal F, Cubero JC. A survey of link prediction in complex networks. ACM Comput Surveys (CSUR). 2017:49(4):69

19. Al Hasan M, Chaoji V, Salem S, Zaki M. Link prediction using supervised learning. In: SDM06: workshop on link analysis, counter-terrorism and security; 2006.

20. Raymond R, Kashima H. Fast and scalable algorithms for semi-supervised link prediction on static and dynamic graphs. In: Joint european conference on machine learning and knowledge discovery in databases. Springer: New York; 2010; p. 131-147.

21. Leroy V, Cambazoglu BB, Bonchi F. Cold start link prediction. In: Proceedings of the 16th ACM SIGKDD international conference on Knowledge discovery and data mining. ACM; 2010; p. 393-402.

22. Wang C, Satuluri V, Parthasarathy S. Local probabilistic models for link prediction. In: Seventh IEEE internationa conference on data mining (ICDM 2007). IEEE: New York; 2007. p. 322-331.

23. Menon AK, Elkan C. Link prediction via matrix factorization. In: Joint european conference on machine learning and knowledge discovery in databases. Springer: New York; 2011. p. 437-452.

24. Fang $Y$, Lin W, Zheng WW, Wu M, Chang KCC, Li XL. Semantic proximity search on graphs with metagraph-based learning. In: 2016 IEEE 32nd International Conference on Data Engineering (ICDE). IEEE; 2016; p. 277-288.

25. Gupta M, Kumar P, Bhasker B. DPRel: a meta-path based relevance measure for mining heterogeneous networks. Information Systems Frontiers. 2017; p. 1-17.

26. Huang Z, Zheng Y, Cheng R, Sun Y, Mamoulis N, Li X. Meta structure: Computing relevance in large heterogeneous information networks. In: Proceedings of the 22nd ACM SIGKDD International Conference on Knowledge Discovery and Data Mining. ACM; 2016; p. 1595-1604.

27. Zhou Y, Huang J, Sun H, Sun Y. Recurrent meta-structure for robust similarity measure in heterogeneous information networks. arXiv preprint. 2017; arXiv:1712.09008.

28. Sun Y, Han J, Yan X, Yu PS, Wu T. Pathsim: Meta path-based top-k similarity search in heterogeneous information networks. Proc VLDB Endowment. 2011;4(11):992-1003.

29. He J, Bailey J, Zhang R. Exploiting transitive similarity and temporal dynamics for similarity search in heterogeneous information networks. In: International Conference on Database Systems for Advanced Applications. Springer: New York; 2014. p. 141-155.

30. Yao K, Mak HF, et al. PathSimExt: revisiting PathSim in heterogeneous information networks. In: International Conference on Web-Age Information Management. Springer: New York; 2014; p. 38-42. 
31. Shi C, Kong X, Huang Y, Philip SY, Wu B. Hetesim: a general framework for relevance measure in heterogeneous networks. IEEE Trans Knowledge Data Eng. 2014;26(10):2479-92.

32. Meng X, Shi C, Li Y, Zhang L, Wu B. Relevance measure in large-scale heterogeneous networks. In: Asia-Pacific Web Conference. Springer: New York; 2014. p. 636-643.

33. Ricci F, Rokach L, Shapira B. In: Ricci F, Rokach L, Shapira B, Kantor PB, editors. Introduction to Recommender Systems Handbook. Boston: Springer US; 2011; p. 1-35. https://doi.org/10.1007/978-0-387-85820-3_1.

34. Basilico J, Hofmann T. Unifying collaborative and content-based filtering. In: Proceedings of the twenty-first international conference on Machine learning; 2004; p. 9.

35. Jafarkarimi H, Sim ATH, Saadatdoost R. A naive recommendation model for large databases. Int J Inform Education Technol. 2012;2(3):216.

36. Liang D, Altosaar J, Charlin L, Blei DM. Factorization meets the item embedding: Regularizing matrix factorization with item co-occurrence. In: Proceedings of the 10th ACM conference on recommender systems; 2016; p. 59-66.

37. Schafer JB, Frankowski D, Herlocker J, Sen S. In: Brusilovsky P, Kobsa A, Nejdl W, editors. Collaborative Filtering Recommender Systems. Berlin, Heidelberg: Springer Berlin Heidelberg; 2007; p. 291-324. https://doi.org/10.1007/ 978-3-540-72079-9_9.

38. Van Meteren R, Van Someren M. Using content-based filtering for recommendation. In: Proceedings of the Machine Learning in the New Information Age: MLnet/ECML2000 Workshop. vol. 30; 2000; p. 47-56.

39. Perozzi B, Al-Rfou R, Skiena S. DeepWalk. Proceedings of the 20th ACM SIGKDD international conference on Knowledge discovery and data mining - KDD '14. 2014; https://doi.org/10.1145/2623330.2623732.

40. Grover A, Leskovec J. node2vec: Scalable feature learning for networks. In: Proceedings of the 22nd ACM SIGKDD international conference on Knowledge discovery and data mining; 2016; p. 855-864.

41. Chang S, Han W, Tang J, Qi GJ, Aggarwal CC, Huang TS. Heterogeneous Network Embedding via Deep Architectures. In: Proceedings of the 21th ACM SIGKDD International Conference on Knowledge Discovery and Data Mining. KDD '15. New York: Association for Computing Machinery; 2015; p. 119-128. https://doi.org/10.1145/2783258.2783296.

42. Xu L, Wei X, Cao J, Yu PS. Embedding of Embedding (EOE): Joint embedding for coupled heterogeneous networks. In: Proceedings of the Tenth ACM International Conference on Web Search and Data Mining. WSDM '17. New York: Association for Computing Machinery; 2017; p. 741-749. https://doi.org/10.1145/3018661.3018723.

43. Dong Y, Chawla NV, Swami A. Metapath2vec: scalable representation learning for heterogeneous networks. In: Proceedings of the 23rd ACM SIGKDD International Conference on Knowledge Discovery and Data Mining. KDD '17. New York: Association for Computing Machinery; 2017; p. 135-144. https://doi.org/10.1145/3097983.3098036.

44. Shi C, Hu B, Zhao WX, Philip SY. Heterogeneous information network embedding for recommendation. IEEE Trans Knowl Data Eng. 2018;31(2):357-70.

45. Xu QS, Liang YZ. Monte Carlo cross validation. Chemometrics Intelligent Laboratory Syst. 2001;56(1):1-11.

46. Arlot S, Celisse A, et al. A survey of cross-validation procedures for model selection. Stat Surveys. 2010;4:40-79.

47. Macskassy SA. On the study of social interactions in twitter. In: Sixth International AAAI Conference on Weblogs and Social Media; 2012.

48. Tang J, Zhang J, Yao L, Li J, Zhang L, Su Z. ArnetMiner: extraction and mining of academic social networks. In: KDD'08; 2008; p. 990-998.

49. Masuda N, Porter MA, Lambiotte R. Random walks and diffusion on networks. Phys Rep. 2017;716:1-58.

50. Latapy M, Viard T, Magnien C. Stream graphs and link streams for the modeling of interactions over time. Social Network Anal Mining. 2018;8(1):61.

51. Petrovic LV, Scholtes I. Counting causal paths in big times series data on networks. CoRR. 2019; arXiv:1905.11287. http://arxiv.org/abs/1905.11287.

\section{Publisher's Note}

Springer Nature remains neutral with regard to jurisdictional claims in published maps and institutional affiliations. 